\title{
Proper Holomorphic Maps in Dimension 2 Extend
}

\author{
Klas Diederich AND Sergey PinchuK
}

Abstract. It is shown, that any proper holomorphic map $f: D \rightarrow$ $D^{\prime}$ between bounded domains $D, D^{\prime} \Subset \mathbb{C}^{2}$ with smooth real-analytic boundaries extends holomorphically to a neighborhood of $\bar{D}$.

\section{Contents}

1. Introduction. . . . . . . . . . . . . . . . . . . . . . . 1089

2. Segre varieties. . . . . . . . . . . . . . . . . . . 1092

3. Proper holomorphic correspondences. . . . . . . . . . . . . . 1094

4. Invariance of Segre varieties for correspondences. . . . . . . . . 1097

5. Further invariance properties of Segre varieties. . . . . . . . . . 1099

6. The inverse of proper correspondences. . . . . . . . . . . . . . 1103

7. Extending correspondences are maps. . . . . . . . . . . . . 1104

8. The possible extension as a correspondence. . . . . . . . . 1110

9. The structure of the boundary. . . . . . . . . . . . . 1113

10. Extension across a dense subset of the boundary. . . . . . . . . 1116

11. Extension across strictly pseudoconvex points. . . . . . . . 1118

12. Final steps for proving Theorem 1.2. . . . . . . . . . . . 1120

References. . . . . . . . . . . . . . . . . 1125

1. Introduction. This article contains another step in the investigation of a question which has been studied since a long time by many authors. Namely, we would like to know the answer to the following:

Conjecture 1.1 Let $D, D^{\prime} \Subset \mathbb{C}^{n}$ be domains with real-analytic smooth boundaries and let $f: D \rightarrow D^{\prime}$ be a proper holomorphic map. Then $f$ extends holomorphically to an open neighborhood of $\bar{D}$.

Partial positive answers have been given in many special situations. For surveys on the subject we refer the reader to [20], [5], [10]. Some major steps which we would like to mention here are: 
(1) $D, D^{\prime}$ strictly pseudoconvex and $f$ biholomorphic, H. Lewy (1977) [22], S. Pinchuk (1975) [24]. (This, actually, also contains the case, where $f$ is only supposed to be proper, because, in this case, $f$ is automatically unbranched, S. Pinchuk (1978) [25].)

(2) $D, D^{\prime}$ are pseudoconvex, $f$ is proper holomorphic, S. Baouendi-H. Jacobowitz-F. Treves (1985) [2], S. Baouendi-S. Bell-L. Rothschild (1988) [1], K. Diederich-J.E.Fornæss (1988) [17] and S. Baouendi-L. Rothschild (1988) [3].

(3) Positive results for the non-pseudoconvex case are, for instance, contained in several articles by K. Diederich-J. E. Fornæss from (1979) [15], (1988) [16] and together with Ye (1994) [18] and by S. Bell (1982) [8] and others.

For a long time, it seemed that the most elegant and best suited method for getting positive answers to the conjecture above would be the one using regularity properties of the Bergman projections of $D$ and $D^{\prime}$, namely, the socalled conditions R and Q of S. Bell (for details, see [7] and [6]). However, since D. Barrett constructed in 1984 [4] his counterexamples of domains $D \Subset \mathbb{C}^{2}$ with smooth real-analytic boundaries (non-pseudoconvex), which do not satisfy condition R (and also not condition Q), it has become clear, that other methods have to be used for treating the conjecture in the non-pseudoconvex case.

Since the analytic method encounters the difficulties mentioned above, it is natural to ask for a more geometric approach. This has been developed in a long series of articles using the so-called Segre varieties which seem to us the natural generalization of the classical reflection principle. We use here the tools worked out by S. Webster [27], K. Diederich-S. Webster [19], K. Diederich-J. E. Fornæss [17], [16], [18]. For several technical details of this method we will refer the reader to these articles.

The main result here is as follows:

Theorem 1.2 Let $D, D^{\prime} \Subset \mathbb{C}^{2}$ be domains with smooth real-analytic boundaries and $f: D \rightarrow D^{\prime}$ a proper holomorphic map. Then $f$ extends holomorphically to an open neighborhood of $\bar{D}$.

\section{Remark.}

(a) It has been proved in [18], that under the hypothesis of the theorem, $f$ extends holomorphically to a neighborhood of $\bar{D}$ if it is supposed to be biholomorphic and known to extend homeomorphically to $\bar{D}$. In this article, we will not use this result. Instead, we will give a complete proof of the theorem which, nevertheless, uses many methods from [18].

(b) Our proof of the theorem also gives immediately local versions of the result in terms of cluster sets. However, because our principal interest here is the global problem, we do not formulate them explicitly. 
The proof of Theorem 1.2 consists of the following two major parts:

(1) We show that $f$ extends as a proper holomorphic correspondence to a suitable neighborhood of any point $a \in \partial D$.

(2) We generalize Theorem 3 from [16] to the case of proper holomorphic maps, namely, we show that any such map $f$, which extends as a proper holomorphic correspondence to a neighborhood of a point $a \in \partial D$, extends holomorphically to a neighborhood of $a$.

Our proof given in this article is independent of the arguments used in [16].

Remark: A fundamental step in our proof is Theorem 7.4. X. Huang [21], using our preliminary notes, gave a version of this for $C R$-mappings.

The structure of this article is as follows.

In Section 2 we remind the reader of the Segre varieties and many of their properties which have been used before in the literature. In Section 3 we clarify basic notions concerning proper holomorphic correspondences. The main result of Section 4 is the invariance of Segre varieties extending proper holomorphic correspondences and in Section 5 we extend this result to the inverses of such correspondences. In Section 6 we, finally, consider the same invariance property for proper holomorphic correspondences extending the inverse of proper maps. All this leads in Section 7 to the first main result, namely, that proper maps which extend as proper holomorphic correspondences even extend as maps.

The rest of the article contains the necessary material for locally extending proper holomorphic maps as correspondences. Namely, in Section 8 we construct the "bricks" for the desired correspondences from Segre varieties and give a criterion under which this construction is applicable. Section 9 presents some material about the structure of real-analytic smooth hypersurfaces of finite type in $\mathbb{C}^{2}$, which is essentially already known from previous work (see [14], [15]). The results from this section are the tools for a new proof of the fact that proper holomorphic maps (in dimension 2) always holomorphically extend through an open dense subset of the set of strictly pseudoconvex points in the boundary (Section 10). Building on this and on the construction from Section 8, we obtain in Section 11 the holomorphic extendability across the whole strictly pseudoconvex part of the boundary. In the last section we also construct, in a step by step fashion, the proper holomorphic correspondences extending $f$ at the weakly pseudoconvex points and at the critical points in the "border". Moreover, during all these constructions we always have to do analogous steps for the (multi-valued) inverse $g=f^{-1}$ of the given proper holomorphic map. (They are not necessary if $f$ is biholomorphic.)

Acknowledgements: The collaboration of the authors on this article has been supported by the Departments of Mathematics of the Universities of Wuppertal 
and Marseille and Indiana University, Bloomington. Further support came from the International Science Foundation, grant RK4300. We would like to thank these institutions.

2. Segre varieties. The essential tool in our proof are geometric invariants associated to $\partial D$, which are called Segre varieties. They have been used in the context of the boundary regularity of biholomorphic mappings by S. Webster in [27] and then in [19], where the reader, who is not familiar with this notion, also can find many basic definitions and properties. Important additional properties were explained in Section 1 of [17]. Finally, we also will have to use additional information collected in [18], Section 2 and Lemma 4.2 (together with property (e) following it). For the convenience of the reader and the clarity of the definitions and notations, we mention in this section all basic properties of Segre varieties that will be needed (mostly without proofs).

Notice that, for any domain $D \Subset \mathbb{C}^{n}$ with smooth real-analytic boundary, there is an open neighborhood $W$ of $\partial D$ and a real-analytic function $r: W \rightarrow \mathbb{R}$ such that $D \cap W=\{z \in W: r(z)<0\}$ and $d r(z) \neq 0$ for all $z \in \partial D$. We call this a global real-analytic defining function of $D$. We denote by $r(z, w)$ the complexification of $r$. It is defined on a suitable neighborhood $V \subset \mathbb{C}^{2 n}$ of the diagonal $\Delta \subset W \times W$ and is holomorphic in $z$ and antiholomorphic in $w$. For points $z \in \mathbb{C}^{n}$ we will sometimes write $z=\left({ }^{\prime} z, z_{n}\right) \in \mathbb{C}^{n-1} \times \mathbb{C}$.

Definition 2.1 Let $z^{0} \in \partial D$ be an arbitrary point. We call standard coordinate system for $z^{0}$ with respect to $\partial D$ any local biholomorphic coordinate system centered at $z^{0}$, such that $z^{0}=0$ and a defining function $r$ can be written with respect to these coordinates in the form

$$
r(z)=2 x_{n}+o(|z|)
$$

A pair of open neighborhoods $U_{1} \Subset U_{2}$ is called a standard pair of neighborhoods of $z^{0}$ if it has the following properties:

(a) With respect to a suitable standard coordinate system for $z^{0}$ one has $U_{2}=^{\prime}$ $U_{2} \times U_{2 n}$ with ' $U_{2}$ an open neighborhood of $0 \in \mathbb{C}^{n-1}$ and $U_{2 n}$ an open neighborhood of 0 on the $z_{n}$-axis;

(b) The complexification $r(z, w)$ is well defined on $U_{2} \times U_{1}$, such that for any $w \in U_{1}$ the set

$$
Q_{w}:=\left\{z \in U_{2}: r(z, w)\right\}=0
$$

is a closed smooth complex-analytic hypersurface, called the Segre variety associated to $w$

(c) $Q_{w}$ can be written as a graph. More precisely, there is a holomorphic function $h_{w}\left({ }^{\prime} z\right)$ on ${ }^{\prime} U_{2}$ (depending antiholomorphically on $w$ ), such that

$$
Q_{w}=\left\{\left({ }^{\prime} z, z_{n}\right) \in U_{2}: z_{n}=h_{w}\left({ }^{\prime} z\right)\right\}
$$


(d) for any $\zeta \in Q_{w}$ we denote by ${ }_{\zeta} Q_{w}$ the germ of $Q_{w}$ at $\zeta$.

Remark. Notice that each point $z^{0} \in \partial D$ admits a family of standard pairs of neighborhoods, such that the corresponding neighborhoods $U_{2}$ form a neighborhood basis of $z^{0}$. Notice, furthermore, that the Segre varieties do not depend on the choice of the defining function $r$.

With the notations of Definition 2.1, the function $h\left({ }^{\prime} z, w\right):=h_{w}\left({ }^{\prime} z\right)$ can be written in the form $h\left({ }^{\prime} z, w\right)=\sum_{j} \lambda_{j}(w)^{\prime} z^{j}$ with antiholomorphic functions $\lambda_{j}(w)$ on $U_{1}$. It has been shown in [19] that, for any given real-analytic $\partial D$, there is an integer $N$ such that, for all $z^{0} \in \partial D$ and any standard pair of neighborhoods $U_{1} \Subset U_{2}$ of $z^{0}$, the coefficients $\left\{\lambda_{j}(\cdot):|j| \leq N\right\}$ uniquely determine $Q_{w}$ (for more details see Section 1 of [17]). This makes it easily possible to define the structure of a complex analytic variety of finite dimension on the family of all Segre varieties, such that the maps

$$
\lambda: U_{1} \ni w \mapsto Q_{w}
$$

are finite antiholomorphic branched coverings (for precise statements and details see [17]). For a fixed point $z^{0} \in \partial D$ and a fixed standard pair of neighborhoods $U_{1} \Subset U_{2}$ of $z^{0}$ we denote the variety of Segre varieties by $\mathcal{S}=\mathcal{S}\left(U_{1}, U_{2}\right)$.

Proposition 2.2 The Segre varieties have the following properties (for (a)(f) see Prop. 2.1 of $[18])$ :

(1) For $z, w \in U_{1}$ one has $z \in Q_{w} \Leftrightarrow w \in Q_{z}$;

(2) $z \in Q_{z} \Leftrightarrow z \in \partial D$;

(3) the sets $A_{w}:=\left\{z \in U_{1}: Q_{z}=Q_{w}\right\}$ are finite (they contain at most as many points as the sheet number of $\lambda$ is);

(4) $z \in A_{w} \Leftrightarrow A_{z}=A_{w}$;

(5) for $w \in \partial D$ one has $A_{w} \subset \partial D$;

(6) $A_{w}=\bigcap\left\{Q_{z}: z \in Q_{w}\right\} \cap U_{1}$;

(7) suppose $D, D^{\prime} \Subset \mathbb{C}^{n}$ are domains with smooth real-analytic boundaries. Suppose, furthermore, that $\left(U_{1}, U_{2}\right)$ resp. $\left(U_{1}^{\prime}, U_{2}^{\prime}\right)$ is a standard pair of neighborhoods for $D$ at $z^{0} \in \partial D$ resp. for $D^{\prime}$ at ${z^{\prime}}^{0} \in \partial D^{\prime}$ and that $f: U_{2} \cap D \rightarrow U_{2}^{\prime} \cap D^{\prime}$ is a holomorphic map which extends in a locally biholomorphic way to a neighborhood $V \subset U_{1}$ of $z^{0}$ such that ${z^{\prime}}^{0}=f\left(z^{0}\right)$. Then $f\left(Q_{w} \cap V\right)=Q_{f(w)}^{\prime} \cap V^{\prime}$ for all $w \in V, V^{\prime}:=f(V)$.

The following fact was proved in Lemma 2.4 of [18]: 
Proposition 2.3 Let $z^{0} \in \partial D$ and fix a suitable standard coordinate system and a standard pair of neighborhoods $U_{1}={ }^{\prime} U_{1} \times U_{1 n}, U_{2}={ }^{\prime} U_{2} \times U_{2 n}$ at $z^{0}$. Define for any $w=\left({ }^{\prime} w^{0}, w_{n}\right) \in U_{1} \backslash \bar{D}$ the set

$$
\Omega_{w}:=\left\{{ }^{\prime} z \in{ }^{\prime} U_{2}:\left({ }^{\prime} z, h_{w}\left({ }^{\prime} z\right)\right) \in D\right\}
$$

Then one has:

(1) For any two points $w^{1}, w^{2} \in U_{1} \backslash \bar{D}$ with the same $\operatorname{Im} w_{n}$-coordinates and the same ' $w$-coordinates, but with $\operatorname{Re} w^{1}<\operatorname{Re} w^{2}$ the relation $\Omega_{w^{1}} \subset \Omega_{w^{2}}$ holds.

(2) There is a $\delta>0$, such that for any ' $w \in \in^{\prime} U_{1}$ the set $U_{1}^{\delta}:=U_{1} \cap\{w=$ $\left.\left({ }^{\prime} w, w_{n}\right) \notin \bar{D}: \operatorname{Re} w_{n}>\delta\right\} \neq \varnothing$ and the set $\Omega_{w}$ is connected for any $w \in U_{1}^{\delta}$.

Remark. One of the technical difficulties in using the Segre varieties for proving Theorem 1.2 lies in the fact that, in general, the set $Q_{w} \cap D$ might have several connected components. Because of this, we sometimes will need statement (2) of Proposition 2.3, and in other situations we will need to pick a special connected component of $Q_{w} \cap D$. This can be done as explained in the following passage.

For any point $w \in W$ with $W$ being a small enough open neighborhood of $\partial D$ one has the following fact: The complex line $\ell_{w}$ through $w$ containing the real line passing through $w$ and orthogonal to $\partial D$ intersects the Segre variety $Q_{w}$ at exactly 1 point ${ }^{s} w$, called the symmetric point to $w$. For $w \in W \backslash \bar{D}$ one always has ${ }^{s} w \in D$. The connected component of $Q_{w} \cap D$ containing ${ }^{s} w$ is denoted by ${ }^{s} Q_{w}$ and called the symmetric component. The following estimate is immediate:

Lemma 2.4 For $w \in W$ one has $d(w, \partial D) \simeq d\left({ }^{s} w, \partial D\right)$ as $d(w, \partial D) \rightarrow 0$.

3. Proper holomorphic correspondences. As explained in the Introduction, Theorem 1.2 will be proved in two essential steps. One part is to show that $f$ extends as a proper correspondence. The other part consists in proving that this implies that $f$ even extends holomorphically. In fact, in the step by step approach to the first part, the second part will already be useful. Therefore, we treat it first in this article.

We introduce at first some notation. Namely, for any domain $D \subset \mathbb{C}^{n}$ we denote by $\widehat{D}$ its envelope of holomorphy which is known always to exist in the category of Riemann domains over $\mathbb{C}^{n}$. Furthermore, we denote for any compact set $K \subset D$ by $\widehat{K}$ its holomorphically convex hull (with respect to $\mathcal{O}(D)$ ) as a compact subset of $\widehat{D}$ ). Finally, we introduce the following: 
Principle of notation: To the notation of all objects introduced in this article for the domain $D$ we add a ${ }^{\prime}$ if we consider them for the domain $D^{\prime}$.

We start with the following observation about the envelope of holomorphy which will be useful several times in the article.

Lemma 3.1 Let $f: D \rightarrow D^{\prime}$ be a proper holomorphic map, $a \in \partial D$, and let $a^{\prime} \in \partial D^{\prime}$ be a point in the cluster set $\operatorname{cl}_{f}(a)$ of a under $f$. Then $a \in \widehat{D}$ if $a^{\prime} \in \widehat{D}^{\prime}$.

Proof. Suppose $a \notin \widehat{D}$. Then, for any $K \Subset D$ we have $d(\widehat{K}, a)>0$, where $d(\cdot, \cdot)$ means the euclidean distance. We choose a sequence $\left(a^{\nu}\right) \subset D$ with $a^{\nu} \rightarrow a$ and $a^{\prime \nu}:=f\left(a^{\nu}\right) \rightarrow a^{\prime}$ and a holomorphic function $h \in \mathcal{O}(D)$ such that $h\left(a^{\nu}\right) \rightarrow$ $\infty$. Since $f$ is proper, there is an algebroid function $h^{\prime}$ on $D^{\prime}$ defined by the relation

$$
h^{\prime}\left(z^{\prime}\right):=h \circ f^{-1}\left(z^{\prime}\right) .
$$

More precisely, there are functions $a_{\mu} \in \mathcal{O}\left(D^{\prime}\right)$ for $\mu=1, \ldots, m$, such that for any $z^{\prime} \in D^{\prime}$ and $w \in \mathbb{C}$ one has $w \in h^{\prime}\left(z^{\prime}\right)$ if and only if

$$
w^{m}+a_{1}\left(z^{\prime}\right) w^{m-1}+\cdots+a_{m}\left(z^{\prime}\right)=0 .
$$

Since $a^{\prime} \in \widehat{D}$ and all the $a_{\mu}$ extend holomorphically to $\widehat{D}^{\prime}, h^{\prime}$ has an algebroid extension to a neighborhood $U^{\prime}$ of $a^{\prime}$. Hence $h^{\prime}$ is uniformly bounded near $a^{\prime}$. This is a contradiction.

We now come to the subject of proper holomorphic correspondences. For clarity we make precise what we mean by saying that $f$ extends locally as a proper holomorphic correspondence.

Definition 3.2 (1) A proper holomorphic correspondence is a closed complex analytic subset $F \subset U \times U^{\prime}$, of pure dimension $n, U, U^{\prime} \in \mathbb{C}^{n}$ open, with the property that the projection $\pi: F \rightarrow U$ is proper. The correspondence $F$ is called irreducible if $F \subset U \times U^{\prime}$ is irreducible as an analytic set.

(2) Let $D, D^{\prime} \Subset \mathbb{C}^{n}$ be domains and $z^{0} \in \partial D$ an arbitrary point. We say that $f$ extends as a proper holomorphic correspondence to a neighborhood $U$ of $z^{0}$ if there is an open set $U^{\prime} \subset \mathbb{C}^{n}$ and an irreducible proper holomorphic correspondence $F \subset U \times U^{\prime}$, such that $F \supset \Gamma_{f} \cap\left\{(D \cap U) \times D^{\prime}\right\}$, where $\Gamma_{f}$ is the graph of $f$. We will use the same way of speaking also for multi-valued maps. 


\section{Remarks.}

(1) A correspondence $F$ assigns to any point $z \in U$ a finite number of image points, namely, the points of the set $\widehat{F}(z):=\pi^{\prime}\left(\pi^{-1}(z)\right) \subset U^{\prime}$, where $\pi^{\prime}$ denotes the projection of $F$ to $U^{\prime}$. Counted with multiplicity, the number of such images is equal to the sheet number $m$ of the branched covering $\pi: F \rightarrow U$. After perturbing the coordinates in the image space in an arbitrarily small way, we may make the following assumptions:

Generic Situation: There is a closed low-dimensional complexanalytic subset $B \subset U$ such that, for all $z \in U \backslash B$, the following holds: if ${z^{\prime}}^{1},{z^{\prime}}^{2} \in \widehat{F}(z),{z^{\prime}}^{1} \neq{z^{\prime}}^{2}$, then ${z^{\prime}}_{k}^{1} \neq{z^{\prime}}_{k}^{2}$ for $k=1, \ldots, n$.

The components of the image points from $\widehat{F}(z)$, written as $z_{k}^{\prime}=f_{k}(z)$ for $k=1, \ldots n$, are algebroid functions on $U$, i.e., they satisfy equations of the form

$$
{z^{\prime}}_{k}^{m}+a_{k 1}(z) z_{k}^{\prime m-1}+\cdots+a_{k m}(z)=0
$$

with holomorphic coefficients $a_{k j}(\cdot) \in \mathcal{O}(U)$. Because of the generic situation and since $F$ is supposed to be irreducible, these polynomials are irreducible over $\mathcal{O}(U)$.

(2) Suppose that $\partial D$ is smooth real-analytic. Then, because of [14], it is minimal (in the sense of Tumanov). Therefore, according to a theorem of Trépreau from [26], there are only the following two possibilities: all functions holomorphic on $D$ near $z^{0}$ extend holomorphically to a neighborhood of $z^{0}$, or all functions holomorphic outside of $\bar{D}$ near $z^{0}$ have this extension property. In the first case, any extension of $f$ as a proper holomorphic correspondence to a neighborhood of $z^{0}$ is necessarily a holomorphic map near $z^{0}$, if $U$ has been chosen small enough. Only in the second case, the sheet number $m$ of the extension can be larger than 1 .

(3) We just want to point out for later use that in the situation where $f$ extends as a proper holomorphic correspondence to a neighborhood $U$ of a point $z^{0} \in \partial D, f$ automatically extends as a holomorphic map through an open dense subset of $U \cap \partial D$.

From Remark (1) we immediately obtain the following elementary observation (see also [16]):

Proposition 3.3 If $f$ extends as a proper holomorphic correspondence to a neighborhood $U$ of a point $z^{0} \in \partial D$, then $f$ extends in a Hölder continuous way to $\bar{D} \cap U$. 
Proof. Notice that the cluster set $\operatorname{cl}_{f}(z)$ of any point $z \in \partial D$ under the map $f$ is always connected. On the other hand, for any $z \in \partial D \cap U$, (3.3) leaves only finitely many possibilities for the image points. Hence, $\mathrm{cl}_{f}(z)$ is a singleton implying the continuous extendability of $f$ to $\bar{D} \cap U$. The Hölder continuity of this extension follows again from the system (3.3).

Remark. If $f: D \rightarrow D^{\prime}$ extends near $z^{0} \in \partial D$ as a proper holomorphic correspondence $F \in U \times U^{\prime}$, then ${z^{\prime}}^{0}:=f\left(z^{0}\right)$ is well defined, as we just saw, and $\left(z^{0}, z^{\prime 0}\right) \in F$. We, therefore, can consider the germ of the correspondence $F$ at $\left(z^{0}, z^{\prime}\right)$, and we may always assume that this germ is irreducible.

The following simple lemma will be very useful to us.

Lemma 3.4 Let $D \Subset \mathbb{C}^{n}$ be a domain with smooth real-analytic boundary, $U$ a connected neighborhood of a point $z^{0} \in \partial D$, and suppose that $F \subset U \times U^{\prime}$ is an irreducible proper holomorphic correspondence. Define $F_{c}:=F \cap((U \backslash \bar{D}) \times$ $\left.U^{\prime}\right)$. If $z^{0} \notin \widehat{D}$ and if $U$ has been chosen small enough, then $F_{c}$ is irreducible.

Proof. We denote the sheet number of $\pi: F \rightarrow U$ by $m$ and may assume that the coordinates in $U^{\prime}$ have been chosen such that we are in a generic situation. Then we have, as in (3.3), irreducible pseudopolynomials of the form

$$
z_{k}^{\prime m}+a_{k 1}(z){z^{\prime}}_{k}^{m-1}+\cdots+a_{k m}(z)=0
$$

over $\mathcal{O}(U)$. Suppose now that $F_{c}$ is reducible. Then $F_{c}$ would have an irreducible component $\tilde{F} \subset(U \backslash \bar{D}) \times U^{\prime}$, such that the sheet number of $\pi: \tilde{F} \rightarrow U \backslash \bar{D}$ is less than $m$. Hence, the polynomials (3.4) would be reducible over $\mathcal{O}(U \backslash \bar{D})$. Because of Trépreaus's theorem and since $z^{0} \notin \widehat{D}$, the decomposition of (3.4) would extend to a decomposition over $\mathcal{O}(U)$ (after possibly shrinking the neighborhood $U)$. This is a contradiction, thus proving that $F_{c}$ is irreducible.

4. Invariance of Segre varieties for correspondences . Let $D, D^{\prime} \Subset \mathbb{C}^{n}$ be domains with smooth real-analytic boundaries, and let $f: D \rightarrow D^{\prime}$ be a proper holomorphic mapping which extends as a correspondence $F$ to a neighborhood of the point $z^{0} \in \partial D$. Choose standard coordinates such that $z^{0}=0, f\left(z^{0}\right)=0$ and standard neighborhoods $U_{2} \ni U_{1} \ni 0$ resp. $U_{2}^{\prime} \ni U_{1}^{\prime} \ni 0$, and denote $U_{1}^{*}:=\left\{w: \bar{w} \in U_{1}\right\}, U_{2}^{*}, U_{j}^{\prime *}, D^{*}, F^{*}, \ldots$ analogously. For $z \in U_{2}$ and $\omega \in U_{1}^{*}$ , we put $\rho(z, \omega):=r(z, \bar{\omega})$ and analogously for $\rho^{\prime}$. We write $\hat{F}$ for the setvalued map given by the correspondence $F$, and may suppose that $\hat{F}\left(U_{1}\right) \subset U_{1}^{\prime}$ and $\hat{F}\left(U_{2}\right) \subset U_{2}^{\prime}$. We then have the following invariance property for the Segre varieties under $\hat{F}$ : 
Theorem 4.1 For all $\left(w, w^{\prime}\right) \in F \cap\left(U_{1} \times U_{1}^{\prime}\right)$, the relation $\hat{F}\left(Q_{w}\right) \subset Q_{w^{\prime}}^{\prime}$ holds.

Remark. Notice that this means: any branch of $\hat{F}$ maps any point from $Q_{w}$ to $Q_{w^{\prime}}^{\prime}$ for any $w^{\prime} \in \hat{F}(w)$.

Before we give a strict proof for this theorem, we mention, for the convenience of the reader, the following argument, not quite complete, explaining why the Theorem should be true. Let

$$
\begin{aligned}
M:=\left\{\left(z, z^{\prime}, \omega, \omega^{\prime}\right) \in U_{2} \times U_{2}^{\prime} \times U_{1}^{*} \times U_{1}^{\prime *}:\right. & \\
& \left.\left(z, z^{\prime}\right) \in F,\left(\omega, \omega^{\prime}\right) \in F^{*}, \rho(z, \omega)=0\right\} .
\end{aligned}
$$

It is enough to show that the function $\rho^{\prime}\left(z^{\prime}, \omega^{\prime}\right)$ vanishes on $M$. In a suitable neighborhood $V$ of a point of biholomorphic extendability of the map $f$ we have $r^{\prime}(f(z))=\alpha(z) r(z)$ with a non-vanishing real-analytic function $\alpha$. After complexification of this identity, we get

$$
\rho^{\prime}\left(f(z), f^{*}(\omega)\right)=\alpha(z, \omega) \rho(z, \omega),
$$

where $f^{*}(\omega)=\overline{f(\bar{\omega})}$. Notice that $\left(z, f(z), \omega, f^{*}(\omega)\right) \in M, z \in V, \omega \in V^{*}$. Therefore, $\rho^{\prime}\left(z^{\prime}, \omega^{\prime}\right)$ vanishes on an open non-empty subset of the complex variety $M$. This implies the desired fact $\rho^{\prime} \mid M \equiv 0$ if $M$ would be known to be irreducible.

The (complete) proof of Theorem 1 will be given in several steps:

Proof of Theorem 4.1. We denote $F_{c}:=F \cap\left[\left(U_{1} \backslash \bar{D}\right) \times U_{1}^{\prime}\right]$.

(1) We suppose at first that $\left(w, w^{\prime}\right) \in F_{c}$. We want to show that $f\left(Q_{w} \cap D\right) \subset$ $Q_{w^{\prime}}^{\prime} \cap D^{\prime}$. Near any point $a \in \partial D \cap U_{1}$ of biholomorphic extendability of the map $f$, we have

$$
f\left({ }{ }_{w} Q_{w}\right) \subset Q_{f(w)}^{\prime} \cap D^{\prime} .
$$

We use Lemma 3.4 together with Proposition 2.3, (2) while traveling with $w$ in $U_{1} \backslash \bar{D}$. Namely, if $w$ moves sufficiently far away from $\partial D$, the variety $Q_{w} \cap D$ becomes connected. Then, because of (4.6), even

$$
f\left(Q_{w} \cap D\right) \subset Q_{w^{\prime}}^{\prime} \cap D^{\prime}
$$

holds for any $w^{\prime}$ with $\left(w, w^{\prime}\right) \in F_{c}$. Moving with $w$ down again preserves this relation, since $F_{c}$ is irreducible.

(2) Next we will show that, for any 2 points in $F$ of the form $\left(w, w^{\prime 1}\right)$ and $\left(w, w^{\prime 2}\right), Q_{w^{\prime 1}}^{\prime}=Q_{w^{\prime 2}}^{\prime}$ necessarily. That is, if $\left(w, w^{\prime 1}\right),\left(w, w^{\prime 2}\right) \in F_{c}$, we get from (4.7) that $Q_{w^{\prime 1}}^{\prime}=Q_{w^{\prime 2}}^{\prime}$. From this, the general claim follows again by analytic continuation because of the irreducibility of $F$. 
(3) We write the multi-valued map $\hat{F}$ in the form $\hat{F}=\left\{f^{1}, \ldots, f^{m}\right\}$ and put $w^{\prime j}:=f^{j}(w)$. We need to show that

$$
f^{k}\left(Q_{w}\right) \subset Q_{w^{\prime j}}^{\prime} \text { for all } k, j .
$$

Consider at first the case $w \in U_{1} \backslash \bar{D}$. Then, from (1), we get for all $z \in D \cap Q_{w}$ that $f(z) \in Q_{w^{\prime j}}^{\prime}$. Hence, by $(2), w^{\prime j} \in Q_{f(z)}^{\prime} \equiv Q_{f^{k}(z)}^{\prime}$. Therefore, $f^{k}(z) \in Q_{w^{\prime j}}^{\prime}$. Together, we have shown that $f^{k}\left(Q_{w}\right) \subset Q_{w^{\prime j}}^{\prime}$. By analytic continuation it follows that (4.8) holds for all $w \in U_{1}$ and for all $k, j$. This proves Theorem 4.1.

As an immediate consequence, we obtain the following result:

Corollary 4.2 There exists a single-valued mapping $\varphi: \mathcal{S} \rightarrow \mathcal{S}^{\prime}$ such that the diagram

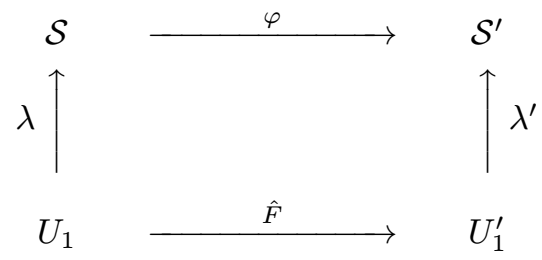

is commutative.

5. Further invariance properties of Segre varieties. Using Theorem 4.1, we will now show the following result:

Theorem 5.1 If the proper holomorphic map $f: D \rightarrow D^{\prime}$ extends as a proper holomorphic correspondence $F \subset U \times U^{\prime}$ to a neighborhood of a point $a \in \partial D$, then $\pi^{\prime}: F \rightarrow U^{\prime}$ also is proper (for suitably chosen sets $U$ and $U^{\prime}$ ).

Remark. In some sense this result can be looked at as saying, that the inverse (multi-valued) map $g:=f^{-1}$ extends as a correspondence to a neighborhood of the point $a^{\prime}:=f(a)$ (which is well-defined, since $F$ gives a continuous extension of $f$ to $D \cup(\partial D \cap U))$. We will have to consider this point of view more precisely and in detail in Section 6.

Proof. We may assume that $a=a^{\prime}=0$. We denote by $\hat{F}$ the multi-valued map given by $F$. It suffices to show that the set $E:=\hat{F}^{-1}(0)$ is discrete. For this, notice that $E$ is analytic. For any point $z \in E \cap D$, one has, according to Theorem 4.1, $Q_{f(z)}^{\prime}=Q_{0}^{\prime}$. Hence $E \cap D$ must be discrete, since $f$ is finiteto-one. But also $\operatorname{dim}(E \cap(U \backslash \bar{D}))=0$, since, again by Theorem 4.1, one has $f\left(Q_{w} \cap D\right) \subset Q_{0}^{\prime} \cap D^{\prime}$ for any $w \in E \backslash \bar{D}$. And finally, because of a well-known 
theorem, no open non-empty part of $E$ can be contained in $\partial D$. Together, this proves the theorem.

Next we want to show the invariance of the Segre varieties under the inverse (multi-valued) map $\hat{G}:=\hat{F}^{-1}: U^{\prime} \rightarrow U$. Of course, this has already been shown (almost) in Theorem 4.1. The only difference is that the underlying map $(f \mid U)^{-1}$ is now itself (possibly) multi-valued.

As a preparation for the proof of this more general invariance property, we introduce at first the so-called reflected points, which are similar to the symmetric points as defined at the end of Section 2. Suppose that $0 \in \partial D$ and $U_{2} \ni U_{1} \ni 0$ are standard neighborhoods. We also fix standard coordinates for $\partial D$ near 0 . We write $\mathbb{C}^{n} \ni w=\left({ }^{\prime} w, w_{n}\right)$, such that ' $w=\left(w_{1}, \ldots, w_{n-1}\right)$. Then, for any $w \in U_{1}$, we define the reflected point ${ }^{\kappa} w$ with respect to $\partial D$ and the coordinates above by the following conditions:

(i) ${ }^{\kappa} w=\left({ }^{\prime} w,{ }^{\kappa} w_{n}\right)$,

(ii) $r\left({ }^{\kappa} w, w\right)=0$, where $r(z)$ is, of course, again a real-analytic defining function for $D$ near 0 and $r(z, w)$ is its complexification, which is holomorphic in $z$ and antiholomorphic in $w$.

The conditions defining ${ }^{\kappa} w$ can also be written in the form

$$
r\left(\left({ }^{\prime} w,{ }^{\kappa} w_{n}\right),\left({ }^{\prime} w, w_{n}\right)\right)=0 .
$$

Notice that the map $\kappa: w \rightarrow{ }^{\kappa} w$ is a real-analytic diffeomorphism depending highly on the choice of the coordinate system. The reality condition for $r$ implies $\kappa \circ \kappa(w) \equiv w$. We have the following result:

Lemma 5.2 Let $E \subset U_{2}$ be a complex analytic set of pure dimension $(n-$ $1)$. Then there exist a standard coordinate system near 0 and a dense open subset $\tilde{E} \subset E$ such that $\kappa(\tilde{E}) \cap E=\varnothing$.

Proof. Let $E=\bigcup_{\nu=1}^{q} E_{\nu}$ be the decomposition of $E$ into irreducible components. Fix points $w^{\nu} \in E_{\nu}$. After a suitable small deformation of the coordinate system, we have $\kappa\left(w^{1}\right) \notin E$. Repeating this argument gives $\kappa\left(w^{\nu}\right) \notin E$ for all $\nu=1, \ldots, q$. Hence $\operatorname{dim}\left(\kappa\left(E_{\nu}\right) \cap E\right)<n-1$ for all $\nu=1, \ldots, q$. Therefore, $\operatorname{dim}(\kappa(E) \cap E)<n-1$. This proves the lemma.

Next we introduce the following notations:

We choose standard neighborhoods $U_{2} \ni U_{1} \ni 0, \tilde{U}_{2} \ni \tilde{U}_{1} \ni 0$ with respect to $0 \in \partial D$ and $U_{2}^{\prime} \ni U_{1}^{\prime} \ni 0$ with respect to $0 \in \partial D^{\prime}$, such that $\hat{F}\left(U_{j}\right) \subset U_{j}^{\prime}$, $\hat{G}\left(U_{j}^{\prime}\right) \subset \tilde{U}_{j}$. Let, furthermore, $m$ (resp. $\left.m^{\prime}\right)$ be the sheet number of $\pi: F \cap\left(U_{1} \times\right.$ $\left.U_{1}^{\prime}\right) \rightarrow U_{1}$ (resp. of $\pi^{\prime}: F \cap\left(\tilde{U}_{1} \times U_{1}^{\prime}\right) \rightarrow U_{1}^{\prime}$ ), and let $\hat{m}$ be the sheet number of 
$f: D \cap U_{1} \rightarrow D^{\prime} \cap U_{1}^{\prime}$ or, more precisely, $\#\left(f^{-1}\left(w^{\prime}\right) \cap \tilde{U}_{1}\right)$ for generic $w^{\prime} \in D^{\prime} \cap U_{1}^{\prime}$. We put $E:=\left\{w \in U_{1}: \# \pi^{-1}(w)<m\right\}, E^{\prime}:=\left\{w^{\prime} \in U_{1}^{\prime}: \# \pi^{\prime-1}\left(w^{\prime}\right)<m^{\prime}\right\}$, $\tilde{F}:=F \backslash \pi^{\prime-1}\left(E^{\prime}\right), F_{c}^{\prime}:=F \cap\left(\tilde{U}_{1} \times\left(U_{1}^{\prime} \backslash \bar{D}^{\prime}\right)\right)$ and $\tilde{F}_{c}^{\prime}:=F_{c}^{\prime} \cap \tilde{F}$.

The generalized invariance property of the Segre varieties can be formulated as follows:

Theorem 5.3 For all $\left(w, w^{\prime}\right) \in F \cap\left(\tilde{U}_{1} \times U_{1}^{\prime}\right)$ one has $\hat{G}\left(Q_{w^{\prime}}^{\prime}\right) \subset Q_{w}$.

For the proof we need the following part of the theorem as a first step:

Lemma 5.4 For all $\left(w, w^{\prime}\right) \in F_{c}^{\prime}$ the relation $g\left(s_{w^{\prime}} Q_{w^{\prime}}^{\prime}\right) \subset Q_{w}$ holds.

\section{Remarks.}

(a) For both, the theorem and the lemma, the underlying situation is the one given in Theorem 5.1. We recommend, that the reader observes while reading the proofs of the Lemma and the Theorem, that no use is being made of the fact that the projection $\pi: F \rightarrow U$ is proper.

(b) We will prove the lemma and the theorem at first only under the following hypothesis.

\section{AdDitional hypothesis: $a^{\prime} \notin \hat{D}$.}

Proof of the Lemma: Notice at first that the branch set $E_{g}^{\prime}$ of $g$ (the set of points $w^{\prime} \in D^{\prime}$, where $\#\left(g\left(w^{\prime}\right)\right)$ is less than it is generically) is contained in $E^{\prime}$. We choose coordinates as in Lemma 5.2.

(1) We show that, for all $\left(w^{0}, w^{0}\right) \in \tilde{F}_{c}^{\prime}$, there exists a branch $g^{1}$ of $g$ in a neighborhood of ${z^{\prime}}^{0}:=\kappa\left({w^{\prime}}^{0}\right)$ such that for $z^{\prime}:=\kappa\left(w^{\prime}\right)$

$$
g^{1}\left({ }_{z^{\prime}} Q_{w^{\prime}}^{\prime}\right) \subset Q_{w}
$$

for all $\left(w, w^{\prime}\right) \in F$ close to $\left(w^{0}, w^{\prime 0}\right)$.

Namely, this is true near any point $\left(a, a^{\prime}\right) \in F$ with $a^{\prime} \in \partial D^{\prime}$ such that some branch $g^{1}$ of $g$ extends biholomorphically and $a=g\left(a^{\prime}\right)$. Connect $\left(a, a^{\prime}\right)$ and $\left(w^{0}, w^{\prime 0}\right)$ by a curve in $\tilde{F}_{c}^{\prime} \cup\left\{\left(a, a^{\prime}\right)\right\}$. This is possible because $F_{c}^{\prime}$ is irreducible, as follows from Lemma 3.4. By analytic continuation we get (5.9).

(2) Let now $g^{1}, \ldots, g^{k}$ be all the branches of $g$ near ${z^{\prime}}^{0}$ for which

$$
g^{\nu}\left({ }_{z^{\prime}} Q_{w^{\prime}}\right) \subset Q_{w}
$$

for all $\left(w, w^{\prime}\right) \in F$ close enough to $\left(w^{0}, w^{\prime 0}\right)$. We will show that the number $k$ does not depend on the choice of $\left(w^{0}, w^{\prime 0}\right)$. 
Namely, we fix $\left(w^{0}, w^{\prime 0}\right)$ as above and take any other point $\left(w^{1}, w^{\prime 1}\right) \in \tilde{F}_{c}^{\prime}$. We connect it to $\left(w^{0}, w^{\prime 0}\right)$ by a curve $\left(w(t), w^{\prime}(t)\right), t \in[0,1]$ in $\tilde{F}_{c}^{\prime}$, such that $z^{\prime}(t):=\kappa\left(w^{\prime}(t)\right) \notin E_{0}^{\prime}$. This can be done because the real codimension of $\kappa\left(E^{\prime}\right)$ in $U_{2}^{\prime}$ is $\geq 2$ and, hence, $\tilde{F}_{c}^{\prime} \backslash \pi^{\prime-1}\left(s\left(E^{\prime}\right)\right)$ is connected.

Let $\tilde{g}^{\nu}$ be an analytic continuation of $g^{\nu}$ along $z^{\prime}(t)$. By analyticity, we have

$$
\tilde{g}^{\nu}\left({ }_{\kappa\left(w^{\prime}\right)} Q_{w^{\prime}}^{\prime}\right) \subset Q_{w} \text { for all } \nu=1, \ldots, k
$$

for all $\left(w, w^{\prime}\right) \in F$ close enough to $\left(w^{1}, w^{\prime 1}\right)$. If $k_{1}$ is the number of all branches of $g$ near $w^{\prime 1}$ satisfying (5.10), then we have $k_{1} \geq k$. But viceversa, one also gets $k_{1} \leq k$. This proves the claim of (2).

(3) Finally, we want to show that $k=\hat{m}$. Suppose that $k<\hat{m}$ and $E_{g}^{\prime}=$ $\bigcup_{\nu=1}^{q} E_{g}^{\prime \nu}$ is the decomposition of $E^{\prime}$ into irreducible components. Then there exist a $\nu$, a point $b^{\prime} \in{E^{\prime}}_{g}^{\nu}$, a point $z^{\prime 0}=\kappa\left(w^{\prime 0}\right) \in\left(U_{1}^{\prime} \cap D^{\prime}\right) \backslash E^{\prime}$ arbitrarily close to $b^{\prime}$, and a $w^{0} \in g\left({w^{\prime}}^{0}\right)$ such that:

(i) if $g^{1}, \ldots, g^{k}$ of $g$ are the branches of $g$ near $z^{\prime 0}$ satisfying the relation analogous to $(5.9)$ near $\left(w^{0}, w^{\prime 0}\right)$, then one of them, say $g^{1}$, goes over by analytic continuation along a small curve $z^{\prime}(t), t \in[0,1]$ around $E_{g}^{\prime \nu}$ near $b^{\prime}$ into a branch $g^{\mu}$ of $g$ with $\mu>k$,

(ii) $\kappa\left(a^{\prime}\right) \notin E^{\prime}$ (Lemma 5.2).

(Condition (i) can be satisfied since the graph of $g$ as analytic set is the same as the graph of $f$ and is, hence, irreducible.)

Put $w^{\prime}(t):=\kappa\left(z^{\prime}(t)\right)$ and let $\left(w(t), w^{\prime}(t)\right)$ be the lifting of $w^{\prime}(t)$ to $\tilde{F}_{c}^{\prime}$ with $w(0)=w^{0}$. Then, by (ii), we have $w(1)=w^{0}$, i.e., this lifting ends where it started, namely at $\left(w^{0}, w^{\prime 0}\right) \in \tilde{F}_{c}^{\prime}$. This is a contradiction of the fact that, by continuation along $z^{\prime}(t)$, we obtained the branch $g^{\mu}$ different from all the $g^{1}, \ldots, g^{k}$. This finishes the proof of Lemma 5.4.

Proof of Theorem 3: Based on Lemma 5.4, the proof of Theorem 5.3 is now the same as the proof of Theorem 4.1, steps (1), (2) and (3).

We should not forget that, so far, we have proved both the theorem and the lemma under only the additional hypothesis, that $a^{\prime} \notin \hat{D}$. Therefore, we still have to consider the case $a^{\prime} \in \hat{D}$.

CASE: $a^{\prime} \in \hat{D}$.

It suffices to just give the following reduction to the previous case: If $a^{\prime} \in \hat{D}$, then $a \in \hat{D}$ too, according to Lemma 3.1. (The coordinates have been chosen such that $a=0=a^{\prime}$.) Hence $f$ extends as a proper holomorphic map to a neighborhood $U \subset U_{1}$ of 0 . The set $U^{\prime}:=f(U)$ is open and $f: U \rightarrow U^{\prime}$ is 
proper for proper choices of $U$ and $U_{1}$. We define $g:=(f \mid U)^{-1}$ (multi-valued). Then we have $g\left(U^{\prime} \cap D^{\prime}\right) \subset U \cap D$, and it also easily follows that $g\left(U^{\prime} \backslash \bar{D}^{\prime}\right) \subset U \backslash \bar{D}$. Namely, suppose there is a point $z^{\prime} \in U^{\prime} \backslash \bar{D}^{\prime}$ with a point $z \in g\left(z^{\prime}\right) \cap(U \cap D)$. Then $f \circ g\left(z^{\prime}\right) \in U^{\prime} \cap D^{\prime}$, as we just observed.

We claim that this observation reduces this case to the previous one. The reason is that, according to the theorem of Trépreau, $a^{\prime}=0$ now lies in the (local) envelope of holomorphy of $U^{\prime} \backslash \bar{D}^{\prime}$, such that we have both the lemma and the theorem if we exchange the role of the insides and the outsides of $D$ (resp. $D^{\prime}$ ). This finishes the proof.

An immediate consequence of Theorem 5.3 is the following statement concerning the map $\varphi$ introduced in Corollary 4.2:

Corollary 5.5 The map $\varphi: \mathcal{S} \rightarrow \mathcal{S}^{\prime}$ as defined in Corollary 2 is one-to-one.

6. The inverse of proper correspondences. In order to make the material more easily comprehensible, we considered in Section 5 only (local) proper holomorphic extensions $F$ of the proper holomorphic map $f: D \rightarrow D^{\prime}$ and their inverses. However, for later use, we need to generalize this also to proper holomorphic correspondences extending the inverse of proper holomorphic maps. We now define precisely what we mean by this, and have to distinguish between the purely local extension of the inverse of $f$ and a somewhat more global situation.

We consider the situation of smooth real-analytic domains $D, D^{\prime} \Subset \mathbb{C}^{n}$ and a proper holomorphic map $f: D \rightarrow D^{\prime}$.

Definition 6.1 We say that the global inverse $g:=f^{-1}$ of the map $f$ extends as a proper holomorphic correspondence to a (connected) neighborhood $U^{\prime}$ of a point $a^{\prime} \in \partial D^{\prime}$, if there is an open set $U \subset \mathbb{C}^{n}$ and a closed complex analytic subset $G \subset U \times U^{\prime}$ of pure dimension n (which may possibly be reducible), such that

(i) $\Gamma_{f} \cap\left\{D \times\left(U^{\prime} \cap D^{\prime}\right)\right\} \subset G$;

(ii) $\pi^{\prime}: G \rightarrow U^{\prime}$ is proper.

From this, we highlight the following:

Definition 6.2 Let $a \in \partial D$ and $a^{\prime} \in \operatorname{cl}_{f}(a) \in \partial D^{\prime}$. We say that $g=$ $f^{-1}$ extends as a proper holomorphic correspondence to a neighborhood of the pair $\left(a, a^{\prime}\right)$ (or that the local inverse of $f$ at the pair $\left(a, a^{\prime}\right)$ extends as a proper holomorphic correspondence) if there are open (connected) neighborhoods $U$ of a and $U^{\prime}$ of $a^{\prime}$ and an irreducible closed complex analytic set $F \subset U \times U^{\prime}$ of pure dimension $n$ such that:

(1) $\Gamma_{f} \cap\left\{(U \cap D) \times\left(U^{\prime} \cap D^{\prime}\right)\right\} \subset F$; 
(2) $\pi^{\prime}: F \rightarrow U^{\prime}$ is proper.

Remark. In a somewhat ambiguous way, we can also say that Definition 1 deals with an extension of all branches of $g=f^{-1}$ as proper holomorphic correspondences to a neighborhood of $a^{\prime}$, whereas in Definition 6.2 we restrict ourselves to extending a local irreducible component of $g$ near the pair $\left(a, a^{\prime}\right)$.

Notice at first that Theorem 5.1 in our new terminology can be reformulated in the following way:

Theorem 6.3 (Reformulation of Theorem 5.1) If the map $f: D \rightarrow D^{\prime}$ extends as a proper holomorphic correspondence to a neighborhood of a $\in \partial D$, then $g=f^{-1}$ extends as a proper holomorphic correspondence to a neighborhood of the pair $(a, f(a))$.

As pointed out already before the proof of Theorem 5.3, no use was made in it of the fact that, in the situation as considered there, the projection $\pi: F \rightarrow U$ is proper. Therefore, the proof actually shows that one also has the following more general invariance property:

Theorem 6.4 Let $a \in \partial D$ and $a^{\prime} \in \mathrm{cl}_{f}(a)$. Suppose that $g=f^{-1}$ extends as a proper holomorphic correspondence $G \subset U \times U^{\prime}$ to a neighborhood of the pair $\left(a, a^{\prime}\right)$ (in the sense of Definition 6.2). Let $\hat{G}: U^{\prime} \rightarrow U$ be the (multi-valued) map given by $G$. Then one has

$$
\hat{G}\left(Q_{w^{\prime}}^{\prime} \cap U^{\prime}\right) \subset Q_{w} \cap U
$$

for all $\left(w, w^{\prime}\right) \in G$.

The same arguments as used in the proof of Theorem 5.1 now give the following result.

Theorem 6.5 In the situation of Theorem 6.4 the map $f$ extends as a proper holomorphic correspondence $F$ to a neighborhood of the point $a \in \partial D$. One can, in fact, choose $F=G$ (as complex analytic sets).

7. Extending correspondences are maps . After we have obtained the complete invariance properties of Segre varieties under proper holomorphic correspondences $F$ which extend the given proper holomorphic map $f: D \rightarrow D^{\prime}$, we can do the necessary steps for showing that $F$ even gives an extension of $f$ as a (single-valued) map.

We show at first this result. 
Proposition 7.1 Let $D, D^{\prime} \Subset \mathbb{C}^{n}$ be domains with smooth real-analytic boundaries and $f: D \rightarrow D^{\prime}$ a proper holomorphic mapping. Suppose that $F$ is a proper holomorphic correspondence extending $f$ near a point $z^{0} \in \partial D$, and put ${z^{\prime}}^{0}:=f\left(z^{0}\right)$. Choose standard coordinates for $D$ and for $D^{\prime}$ near $z^{0}$ (resp. ${z^{\prime}}^{0}$ ) and corresponding standard pairs of neighborhoods $U_{2} \ni U_{1} \ni 0$ (resp. $\left.U_{2}^{\prime} \ni U_{1}^{\prime} \ni 0\right)$, such that $\hat{F}\left(U_{1}\right) \subset U_{1}^{\prime}$. Then one has the following:
(a) $\hat{F}\left(\partial D \cap U_{1}\right) \subset \partial D^{\prime} \cap U_{1}^{\prime}$,
(b) $\hat{F}\left(U_{1} \backslash \bar{D}\right) \subset U_{1}^{\prime} \backslash \bar{D}^{\prime}$,
(c) $\hat{F}\left(U_{1} \cap D\right) \subset U_{1}^{\prime} \cap D^{\prime}$.

Proof. (1) To prove (a), we choose $w \in \partial D \cap U_{1}$ and $w^{\prime} \in \hat{F}(w)$. Then, according to Theorem 4.1, $Q_{w^{\prime}}^{\prime}=Q_{f(w)}^{\prime}$. Hence, since $f(w) \in \partial D^{\prime}$, also $w^{\prime} \in$ $\partial D^{\prime}$.

(2) Let $w \in U_{1} \backslash \bar{D}$ be any point, and choose a point $a \in \partial D \cap U_{1}$ of biholomorphic extendability of $f$. Connect $a$ and $w$ by a curve $w(t)$ in $\left(U_{1} \backslash \bar{D}\right) \cup$ $\{a\}$. If $\hat{F}(w) \ni w^{\prime} \notin U_{1}^{\prime} \backslash \bar{D}^{\prime}$, then there is a point $w^{0}=w\left(t_{0}\right) \in U_{1} \backslash \bar{D}$ such that $\hat{F}\left(w^{0}\right) \ni w^{\prime 0} \in \partial D^{\prime}, w^{\prime 0}=f\left(z^{0}\right)$ with $z^{0} \in \partial D$. By Theorem 5.3 we have $Q_{w^{0}}=Q_{z^{0}}$, a contradiction.

(3) The proof of part (c) follows from (a), (b), and the irreducibility of $F_{c}^{\prime}$ as defined before Theorem 5.3. Namely, let us examine first $\left(w^{0}, w^{\prime 0}\right) \in F \cap\left(\left(U_{1} \cap\right.\right.$ $\left.D) \times\left(U_{1}^{\prime} \backslash \bar{D}^{\prime}\right)\right)$. By (b) there is a point $\left(w^{1}, w^{\prime 1}\right) \in F \cap\left(\left(U_{1} \backslash \bar{D}\right) \times\left(U_{1}^{\prime} \backslash \bar{D}^{\prime}\right)\right)$. We connect it by a curve in $F_{c}^{\prime}$ to $\left(w^{0}, w^{\prime 0}\right)$. On this curve there is a point $\left(w^{2},{w^{\prime}}^{2}\right) \in F \cap\left(\left(\partial D \cap U_{1}\right) \times\left(U_{1}^{\prime} \backslash \bar{D}^{\prime}\right)\right)$. This, however, contradicts (a).

The second case, in which there might be a point $\left(w^{0},{w^{\prime}}^{0}\right) \in F \cap\left(\left(U_{1} \cap D\right) \times\right.$ $\left.\left(U_{1}^{\prime} \cap \partial D^{\prime}\right)\right)$, leads to a contradiction in a quite similar way.

As an application of the invariance of the Segre varieties under the extending correspondence and its inverse (Theorems 4.1 and 5.3), we obtain immediately an extension result for the "normal" components of the mappings in suitable coordinates. These are introduced in the following way. Suppose $0 \in \partial D$. It is well known that coordinates near 0 and a defining function $r$ of $D$ near 0 can be chosen in such a way that $r$ has the form

$$
r(z)=2 x_{n}+\sum_{\nu=0}^{\infty} a_{\nu}\left({ }^{\prime} z\right) y_{n}^{\nu},
$$

where the functions $a_{\nu}\left({ }^{\prime} z\right)$ are real-analytic and their complexifications $a_{\nu}\left({ }^{\prime} z,{ }^{\prime} \omega\right)$, which are holomorphic in ${ }^{\prime} z$ and in ' $\omega$, satisfy

$$
a_{\nu}\left({ }^{\prime} z, 0\right)=a_{\nu}\left(0,{ }^{\prime} \omega\right)=0 .
$$


We will call such coordinates "normal" at 0 with respect to $D$. The holomorphic complexification $\rho(z, \omega)$ of $r$ is then given by

$$
\rho(z, \omega)=z_{n}+\omega_{n}+\sum_{\nu=0}^{\infty} a_{\nu}\left({ }^{\prime} z,^{\prime} \omega\right)\left(\frac{z_{n}-\omega_{n}}{2 i}\right)^{\nu} .
$$

Of course, we can make the analogous choices at $0 \in \partial D^{\prime}$ with respect to $D^{\prime}$. We can now formulate the following result.

Proposition 7.2 Suppose that normal coordinates have been chosen for $0 \in \partial D$ with respect to $D$ and for $0 \in \partial D^{\prime}$ with respect to $D^{\prime}$. Denote by $f_{n}, g_{n}$, $\hat{F}_{n}$, and $\hat{G}_{n}$ the $n^{\text {th }}$-component of the (multi-valued) mappings $f, g, \hat{F}$, and $\hat{G}$. Then there are neighborhoods $U$ and $U^{\prime}$ of 0 such that one has:

(a) The components $\hat{F}_{n}$ and $\hat{G}_{n}$ are single-valued on $U$ (resp. on $\left.U^{\prime}\right), f_{n} \in$ $\mathcal{O}(U)$ and $g_{n} \in \mathcal{O}\left(U^{\prime}\right)$.

(b) There are functions $a \in \mathcal{O}(U)$ and $b \in \mathcal{O}\left(U^{\prime}\right)$ with $a(0) \neq 0$ and $b(0) \neq 0$ such that

$$
f_{n}\left({ }^{\prime} z, z_{n}\right)=z_{n} a\left({ }^{\prime} z, z_{n}\right), \quad g_{n}\left({ }^{\prime} z^{\prime}, z_{n}^{\prime}\right)=z_{n}^{\prime} b\left({ }^{\prime} z,{ }^{\prime} z_{n}^{\prime}\right)
$$

Proof. (1) We choose with respect to the given normal coordinates defining functions $r$ of $D$ near 0 and $r^{\prime}$ of $D^{\prime}$ near 0 , such that (7.11) holds and, analogously,

$$
r^{\prime}\left(z^{\prime}\right)=2 x_{n}^{\prime}+\sum_{\nu=0}^{\infty} a_{\nu}^{\prime}\left({ }^{\prime} z^{\prime}\right) y_{n}^{\prime \nu} .
$$

By the implicit function theorem, the Segre varieties $Q_{\bar{\omega}}$ and $Q_{\overline{\omega^{\prime}}}^{\prime}$ are then given by equations of the form

$$
\begin{aligned}
& z_{n}=-\omega_{n}+\sum_{|\nu|>0} b_{\nu}(\omega)^{\prime} z^{\nu} \\
& z_{n}^{\prime}=-\omega_{n}^{\prime}+\sum_{|\nu|>0} b_{\nu}^{\prime}\left(\omega^{\prime}\right) z^{\prime \nu}
\end{aligned}
$$

respectively. Therefore, $Q_{w^{1}}=Q_{w^{2}}$ implies $w_{n}^{1}=w_{n}^{2}$ and $Q_{w^{\prime 1}}^{\prime}=Q_{w^{\prime 2}}^{\prime}$ implies ${w^{\prime}}_{n}^{1}={w^{\prime}}_{n}^{2}$. Because of Theorem 4.1, however, this means that for any $w \in U$, where $U$ is a small enough neighborhood of 0 , and any two points $w^{\prime 1}, w^{\prime 2} \in$ $\hat{F}(w)$, one has $\hat{F}\left(Q_{w}\right) \subset Q_{w^{\prime 1}}^{\prime}$ and also $\hat{F}\left(Q_{w}\right) \subset Q_{w^{\prime 2}}^{\prime}$. Hence, $Q_{w^{\prime 1}}^{\prime}=Q_{w^{\prime 2}}^{\prime}$ and, therefore, $w_{n}^{\prime 1}=w_{n}^{\prime 2}$. This shows that $\hat{F}_{n}$ is single-valued on $U$. In particular, it gives the desired holomorphic extension of $f_{n}$ to $U$. By using Theorem 5.3, we obtain the respective facts for $\hat{G}_{n}$ and $g_{n}$. 
(2) Because of (7.15), one has $Q_{0}=\left\{z_{n}=0\right\}$ and $Q_{0}^{\prime}=\left\{z_{n}^{\prime}=0\right\}$. Together with Theorems 4.1 and 5.3, this means that

$$
\begin{array}{r}
f_{n}\left({ }^{\prime} z, z_{n}\right)=0 \\
g_{n}\left({ }^{\prime} z^{\prime}, z_{n}^{\prime}\right)=0
\end{array} \Leftrightarrow \quad \begin{aligned}
& z_{n}=0 \\
& z_{n}^{\prime}=0
\end{aligned}
$$

Therefore,

$$
\begin{aligned}
f_{n}\left({ }^{\prime} z, z_{n}\right) & =z_{n}^{p} \tilde{f}_{n}\left({ }^{\prime} z, z_{n}\right) \\
g_{n}\left({ }^{\prime} z^{\prime}, z_{n}^{\prime}\right) & =z^{\prime}{ }_{n}^{\prime} \tilde{g}_{n}\left({ }^{\prime} z^{\prime}, z_{n}^{\prime}\right)
\end{aligned}
$$

where $p, q \in \mathbb{N}, \tilde{f}_{n}(0) \neq 0$ and $\tilde{g}_{n}(0) \neq 0$. From $f \circ \hat{G}=i d$ we get

$$
\begin{aligned}
z_{n}^{\prime} & =z_{n}^{p} \tilde{f}_{n}\left({ }^{\prime} z, z_{n}\right)=\left[z^{\prime q}{ }_{n} \tilde{g}_{n}\left({ }^{\prime} z^{\prime}, z_{n}^{\prime}\right)\right]^{p} \tilde{f}_{n}\left({ }^{\prime} g\left(z^{\prime}\right), g_{n}\left(z^{\prime}\right)\right) \\
& =z^{\prime p q}{ }_{n}^{p q} \tilde{g}_{n}\left({ }^{\prime} z^{\prime}, z_{n}^{\prime}\right)^{p} \tilde{f}_{n}\left(g\left(z^{\prime}\right)\right)
\end{aligned}
$$

Since $\tilde{g}_{n}(0) \neq 0$ and $\tilde{f}_{n}(0) \neq 0$, we have $p q=1$, hence $p=1$ and $q=1$. This finishes the proof.

The decisive information, which will allow us to prove that $f: D \rightarrow D^{\prime}$ extends as a map if it extends as a proper holomorphic correspondence, consists in showing that the critical set $E^{\prime}$ of the Segre map $\lambda^{\prime}: U_{2}^{\prime} \rightarrow \mathcal{S}^{\prime}$, i. e., the set of points where $\lambda^{\prime}$ is not locally one-to-one, is closely related to the branching locus $E \subset U_{2}$ of $\hat{F}$ and enters into the domain $D^{\prime}$ in a nice way. In this article, we will show this only for the case $D, D^{\prime} \Subset \mathbb{C}^{2}$. The general case, which is considerably more complicated, will be treated by the authors in a forthcoming article. (Notice that $E \subset U_{2}$ and $E^{\prime} \subset U_{2}^{\prime}$ are always complex analytic sets of pure dimension 1.)

Theorem 7.3 (a) One has $\hat{F}(E) \subset E^{\prime}$.

(b) Let $E^{\prime}=\bigcup E_{\nu}^{\prime}$ be the decomposition of $E^{\prime}$ into irreducible components in $U_{2}^{\prime}$. Then , any $E_{\nu}^{\prime}$ with $0 \in E_{\nu}^{\prime}$ enters into $D^{\prime}$. Furthermore, the estimate

$$
\left|z_{1}^{\prime}\right|=o\left(\left|z_{2}^{\prime}\right|^{1 / p}\right)
$$

holds on $E^{\prime}$, where $p$ denotes the 1-type of $\partial D^{\prime}$ at 0 .

Proof. To show (a), we just observe that we know, from Corollary 4.2, that $\lambda^{\prime} \circ \hat{F}=\varphi \circ \lambda$. Since $\varphi \circ \lambda$ is single-valued, Claim (a) follows.

In order to show (b), we fix a component $E_{\nu}^{\prime}$ with $0 \in E_{\nu}^{\prime}$ and call it, for the time being, $E^{\prime}$. We only have to consider the case $E^{\prime} \neq\left\{w_{1}^{\prime}=0\right\}$. 
(1) We first show how to reduce the claim to the situation where $E^{\prime}$ is a complex manifold of dimension 1 near 0 . The projection $\pi: E^{\prime} \cap\left\{\left|w_{1}^{\prime}\right|<\right.$ $\delta\} \rightarrow\left\{\left|w_{1}^{\prime}\right|<\delta\right\}$ is proper. We call its sheet number $\ell$. Then there is a holomorphic function $h(\zeta)$ on the disc $\left\{|\zeta|<\delta^{1 / \ell}\right\}$ such that

$$
E^{\prime}=\left\{\left(w_{1}^{\prime}, w_{2}^{\prime}\right):\left|w_{1}^{\prime}\right|<\delta, w_{2}^{\prime}=h\left(w_{1}^{\prime / \ell}\right)\right\} .
$$

After the proper holomorphic change of variables,

$$
w_{1}^{\prime}=\tilde{w}_{1}^{\ell}, \quad w_{2}^{\prime}=\tilde{w}_{2}^{\prime},
$$

$E^{\prime}$ becomes a complex manifold (and the new domain $D^{\prime}$ still has realanalytic smooth boundary). Notice that it suffices to prove the theorem in the $\tilde{w}^{\prime}$-coordinates. This finishes (1).

For the sake of simplicity, we again denote the changed coordinates by $w^{\prime}$. After a biholomorphic change, they become normal coordinates with an associate defining function $r^{\prime}\left(z^{\prime}\right)$ for $D^{\prime}$ near 0 of the form (7.11) and (7.12). Since $D^{\prime}$ is of finite type, one has $a_{0}^{\prime} \neq 0$. In fact, $a_{0}^{\prime}\left(z_{1}^{\prime}, \overline{z_{1}^{\prime}}\right)=P_{p}\left(z_{1}^{\prime}, \overline{z_{1}^{\prime}}\right)+o\left(\left|z_{1}^{\prime}\right|^{p}\right)$, where

$$
P_{p}\left(z_{1}^{\prime}, \overline{z_{1}^{\prime}}\right)=\sum_{k=1}^{p-1} \alpha_{k}{z_{1}^{\prime k}}_{z_{1}^{\prime}}^{p-k},
$$

and there exists an index $m: 1 \leq m \leq p-1$ such that $\alpha_{m} \neq 0$. By the implicit function theorem, we can solve the equation

$$
0=\rho^{\prime}\left(z^{\prime}, \omega^{\prime}\right)=z_{2}^{\prime}+\omega_{2}^{\prime}+\sum_{\nu=0}^{\infty} a_{\nu}\left(z_{1}^{\prime}, \omega_{1}^{\prime}\right)\left(\frac{z_{2}^{\prime}-\omega_{2}^{\prime}}{2 i}\right)^{\nu}
$$

for $z_{2}^{\prime}$. We get

$$
z_{2}^{\prime}=-\omega_{2}^{\prime}+\sum_{\nu=1}^{\infty} b_{\nu}\left(\omega^{\prime}\right) z_{1}^{\prime \nu}
$$

Moreover,

$$
b_{m}\left(\omega^{\prime}\right)=-\alpha_{m} \omega_{1}^{\prime p-m}+o\left(\omega_{1}^{p-m}\right)+\sum_{k=1}^{\infty} \beta_{k}\left(\omega_{1}^{\prime}\right) \omega_{2}^{\prime k}
$$

The coefficients $\left\{b_{\nu}\left(\omega^{\prime}\right)\right\}$ together with $-\omega_{2}^{\prime}$ are the components of the map $\lambda^{\prime}$. Furthermore, for any $w \in E^{\prime}$, the map $\lambda^{\prime}$ does not have maximal rank. Hence, $w=\bar{\omega} \in E^{\prime}$ implies $\left(\partial b_{\nu} / \partial \omega_{1}^{\prime}\right)\left(\omega^{\prime}\right)=0$ for any $\nu$. The equation $\left(\partial b_{m} / \partial \omega_{1}^{\prime}\right)\left(\omega^{\prime}\right)=$ 0 means that

$$
\sum_{k=1}^{\infty} \frac{\partial \beta_{k}}{\partial \omega_{1}^{\prime}}\left(\omega_{1}^{\prime}\right) \omega_{2}^{\prime k}=(p-m) \alpha_{m} \omega_{1}^{\prime p-m-1}+o\left(\left|\omega_{1}^{\prime}\right|^{p-m-1}\right)
$$


We know from (7.16), that $w^{\prime} \in E^{\prime}$ is equivalent to $w_{2}^{\prime}=h\left(w_{1}^{\prime}\right)$, and this can be written as $\omega_{2}^{\prime}=\overline{h\left(\overline{\omega_{1}^{\prime}}\right)}=: h^{*}\left(\omega_{1}\right)$. Since $(7.20)$ holds on all of $E^{\prime}$, we can substitute $h^{*}\left(\omega_{1}\right)$ for $\omega_{2}^{\prime k}$ on its left-hand side. The right-hand side shows that the function of $\omega_{1}^{\prime}$ that we obtain then vanishes at 0 exactly with order $p-m-1$. Hence, the order of vanishing of $h$ at the origin must be $\leq(p-m-1)<(p-1)$. The restriction of the defining function $r^{\prime}\left(z^{\prime}\right)$ to $E^{\prime}$ has the form

$$
r^{\prime}\left(z^{\prime}\right) \mid E^{\prime}=2 \operatorname{Re} h\left(z_{1}^{\prime}\right)+O\left(\left|z_{1}^{\prime}\right|^{p}\right) .
$$

Since $\operatorname{Re} h\left(z_{1}^{\prime}\right)$ vanishes with order less than $p$, as a harmonic term changes sign, $\rho^{\prime}$ also changes sign on $E^{\prime}$. This finishes the proof of the theorem.

We now are ready to show the following result.

Theorem 7.4 Let $D, D^{\prime} \Subset \mathbb{C}^{2}$ be domains with smooth real-analytic boundaries and $f: D \rightarrow D^{\prime}$ a proper holomorphic map which extends as a proper holomorphic correspondence $F$ to a neighborhood of $z^{0} \in \partial D$. Then $f$ extends as a holomorphic map to a neighborhood of $z^{0}$.

Proof. We may suppose that $z^{0}=0$ and ${z^{\prime}}^{0}:=f\left(z^{0}\right)=0$. We use all the standard notations introduced above and choose normal coordinates for $D$ and for $D^{\prime}$ near 0 . We fix a small constant $c>0$ and put for any $\varepsilon \geq 0$

$$
\Delta_{\varepsilon}^{\prime}:=\left\{\left(z_{1}^{\prime}, z_{2}^{\prime}\right):\left|z_{1}^{\prime}\right|<c, z_{2}^{\prime}=-\varepsilon\right\}
$$

By Proposition $7.2, \hat{F}^{-1}\left(\Delta_{\varepsilon}^{\prime}\right)$ is a complex manifold (near 0 ) given by the equation $z_{2} \tilde{f}(z)=-\varepsilon$. We may choose a neighborhood $U=V_{1} \times V_{2} \ni 0$, where $V_{1}$ and $V_{2}$ are small discs in $\mathbb{C}$, such that for all $\varepsilon \in\left(0, \varepsilon_{0}\right), \varepsilon_{0}>0$ small enough, the set $\Delta_{\varepsilon}:=\hat{F}^{-1}\left(\Delta_{\varepsilon}^{\prime}\right) \cap U$ is a graph of the form $\left\{z_{2}=h_{\varepsilon}\left(z_{1}\right): z_{1} \in V_{1}\right\}$. Notice that, because of Propositions 7.1 and $7.2, \hat{F}^{-1}((0,-\varepsilon)) \subset D$. We show:

\section{Claim}

(a) For any $\varepsilon \in\left(0, \varepsilon_{0}\right)$, the map $f$ extends holomorphically along any curve in $\Delta_{\varepsilon}$ to a map $f_{\varepsilon}$, holomorphic in a neighborhood $U_{\varepsilon}$ of $\overline{\Delta_{\varepsilon}}$ in $\mathbb{C}^{2}$. Moreover,

(b) there is a $\delta>0$ such that $U_{\varepsilon}$ contains a $\delta$-neighborhood of $\partial \Delta_{\varepsilon}$ for any $\varepsilon \in\left(0, \varepsilon_{0}\right)$.

Let us first prove (a). The multi-valued map $\hat{F}$ branches on $E \subset \hat{F}^{-1}\left(E^{\prime}\right)$ and, according to Theorem 7.3, one has $\Delta_{\varepsilon}^{\prime} \cap E^{\prime} \subset D^{\prime}$. Hence, because of Proposition 7.1, $\hat{F} \mid \Delta_{\varepsilon}$ can branch only in $\Delta_{\varepsilon} \cap D$. Therefore, the map $f$ analytically extends along each path in $\Delta_{\varepsilon}$. By the monodromy theorem, this gives a holomorphic extension of $f$ to a neighborhood of $\overline{\Delta_{\varepsilon}}$. 
In order to show that (b) also holds, we observe that, because of part( $b$ ) of Theorem 7.3, we have $\overline{\Delta_{0}^{\prime}} \cap E^{\prime}=\{0\}$. From part (a) of the same theorem together with Theorem 5.1, it follows that $\overline{\Delta_{0}} \cap E=\{0\}$. Hence there exists a $\delta>0$ such that the $2 \delta$-neighborhood $W$ of $\partial \Delta_{0}$ does not intersect $E$. Hence (b) follows by continuity.

The theorem follows from the claim by the disc theorem applied to the family of the $f_{\varepsilon}$ and the fact that $0 \in \Delta_{0}$.

8. The possible extension as a correspondence. In this section we start with the constructions that, finally, will show that the map $f: D \rightarrow D^{\prime}$ under the conditions of Theorem 1.2 extends as a proper holomorphic correspondence to a suitable neighborhood of any point $z^{0} \in \partial D$. This result has already been proved in [18], Lemma 4.1, under the additional hypothesis that $f$ is already known to extend homeomorphically to a map from $\bar{D}$ to $\bar{D}^{\prime}$. Although the basic techniques here are the same as in [18], our proof is (logically) independent of Lemma 4.1 from [18] and, therefore, it also is an alternative proof for this lemma.

The desired correspondences will be built from Segre varieties. The pertinent part is as follows.

Definition 8.1 (1) For any point $z^{0} \in \partial D$ with a standard coordinate system and a standard pair of neighborhoods $U_{1} \Subset U_{2}$ and a suitably small open neighborhood $U^{\prime}$ of $\partial D^{\prime}$, we define

$$
V:=\left\{\left(w, w^{\prime}\right) \in\left(U_{1} \backslash \bar{D}\right) \times\left(U^{\prime} \backslash \overline{D^{\prime}}\right): f\left(Q_{w} \cap D\right) \supset{ }^{s} w^{\prime} Q_{w^{\prime}}^{\prime}\right\}
$$

and call it the Segre correspondence for $f$ on $U_{1} \backslash \bar{D}$. By $\pi, \pi^{\prime}$ we denote the natural projections from $\mathbb{C}_{w}^{2} \times \mathbb{C}_{w^{\prime}}^{2}$ to $\mathbb{C}_{w}^{2}, \mathbb{C}_{w^{\prime}}^{2}$, respectively, and put

$$
E:=\left\{z \in D \cap U_{2}: z \in f^{-1}\left({ }^{s} w^{\prime}\right) \cap Q_{w} \text { for some }\left(w, w^{\prime}\right) \in V\right\}
$$

(2) For the global inverse $g=f^{-1}$ of $f$, any point $z^{\prime 0} \in \partial D^{\prime}$ with a standard pair of neighborhoods ${z^{\prime}}^{0} \in U_{1}^{\prime} \Subset U_{2}^{\prime}$ and a suitably small neighborhood $U$ of $\partial D$, we put

$$
V^{\prime}:=\left\{\left(w, w^{\prime}\right) \in(U \backslash \bar{D}) \times\left(U_{1}^{\prime} \backslash \overline{D^{\prime}}\right): g\left(Q_{w^{\prime}}^{\prime} \cap D^{\prime}\right) \supset{ }^{s}{ }_{w} Q_{w}\right\}
$$

and

$$
E^{\prime}:=\left\{z^{\prime} \in D^{\prime} \cap U_{2}^{\prime}: z^{\prime}=f\left({ }^{s} w\right) \text { for some } w \in \pi\left(V^{\prime}\right)\right\}
$$

\section{Remarks.}


(1) Notice that this definition indeed makes sense as soon as $U^{\prime}$ and $U$ have been chosen small enough, because then $\bar{U}^{\prime}$ (resp. $\bar{U}$ ) can be covered by a finite number of standard coordinate systems and neighborhoods $U_{1}^{\prime}$ (resp $U_{1}$ ) of standard pairs of neighborhoods $U_{1}^{\prime} \Subset U_{2}^{\prime}$ (resp. $U_{1} \Subset U_{2}$ ). It easily follows from the properties of the Segre varieties that $V$ and $V^{\prime}$ do not depend on these choices.

(2) The condition defining $V^{\prime}$ is equivalent to the condition $f\left(s_{w} Q_{w}\right) \subset Q_{w^{\prime}}^{\prime}$.

(3) The definition of $V^{\prime}$ from (8.23) is symmetric to the definition of $V$ from (8.21). In the following, it will be quite often necessary to consider such symmetric situations, and to prove symmetric statements. We would like to point out to the reader that he can always ignore the respective second cases if he first wants to understand how the proof of Theorem 1.2 works in the case of biholomorphic maps $f: D \rightarrow D^{\prime}$.

A fundamental fact for the use of the Segre correspondences for extending $\Gamma_{f}$ is now contained in the following two lemmas, which give conditions under which the sets $V$ (resp. $V^{\prime}$ ) from above are complex-analytic varieties.

Lemma 8.2 If in the situation of Definition 8.1 the set $E$ is relatively compact in $U_{2}$, then:

(a) $V$ is complex-analytic in $\left(U_{1} \backslash \bar{D}\right) \times\left(U^{\prime} \backslash \bar{D}^{\prime}\right)$;

(b) there are no limit points of $V$ on $\left(U_{1} \backslash \bar{D}\right) \times \partial U^{\prime}$;

(c) if $\Sigma^{\prime} \subset \partial D^{\prime}$ denotes the set of points, where the global inverse $g:=f^{-1}$ extends as a proper holomorphic correspondence, then $V$ does not have any limit point on $\left(U_{1} \backslash \bar{D}\right) \times \Sigma^{\prime}$.

Proof. (a) Let $\left(w^{0}, w^{\prime 0}\right) \in V$. Notice that, because of Proposition 2.3, for a small enough neighborhood $W \subset \mathbb{C}^{4}$ of $\left(w^{0}, w^{\prime^{0}}\right)$, a suitable standard pair of neighborhoods $U_{1}^{\prime} \Subset U_{2}^{\prime}$ with $w^{\prime 0} \in U_{1}^{\prime}$ and any $\left(w, w^{\prime}\right) \in W$, the condition

$$
f\left(Q_{w} \cap D\right) \supset{ }^{s} w^{\prime} Q_{w^{\prime}}^{\prime}
$$

is equivalent to the condition

$$
f\left(Q_{w} \cap D\right) \supset \tilde{w}^{\prime} Q_{w^{\prime}}^{\prime},
$$

where $\tilde{w}^{\prime}$ is the unique point on $Q_{w^{\prime}}^{\prime}$ with $\tilde{w}_{1}^{\prime}={ }^{s}{w^{\prime}}_{1}^{0}$. It is easy to see that condition (8.25) is equivalent to a system of holomorphic equations. This shows that $V$ is locally complex-analytic. We still have to show that $V$ is closed in $\left(U_{1} \backslash \bar{D}\right) \times\left(U^{\prime} \backslash \bar{D}^{\prime}\right)$. To this end, let $\left(w^{\nu}, w^{\prime \nu}\right) \in V$ be a sequence converging to a point $\left(w^{0}, w^{\prime 0}\right) \in\left(U_{1} \backslash \bar{D}\right) \times\left(U^{\prime} \backslash \bar{D}^{\prime}\right)$. We have to show that $\left(w^{0}, w^{\prime 0}\right) \in V$. 
Since $w^{\prime \nu} \rightarrow{w^{\prime}}^{0},{ }^{s} w^{\prime \nu} \rightarrow{ }^{s}{w^{\prime}}^{0} \in D^{\prime}$ too, and, hence, according to the definition of $V$, there exist points $\zeta^{\nu} \in f^{-1}\left({ }^{s} w^{\prime \nu}\right) \cap Q_{w^{\nu}}$ such that $\zeta^{\nu} \rightarrow \zeta^{0} \in$ $f^{-1}\left({ }^{s}{w^{\prime}}^{0}\right) \subset D$. By the continuity of the Segre varieties and the compactness assumption on $E$, it follows that $\zeta^{0} \in Q_{w^{0}} \cap U_{2}$, and, hence, $f\left(Q_{w^{0}} \cap D\right) \supset_{s_{w^{\prime}}}$ $Q_{w^{\prime}}^{\prime}$. This finishes the proof of (a).

(b) Put $\delta:=d\left(\partial U^{\prime}, \partial D^{\prime}\right)>0$. Because of Lemma 2.4, there exists a $\delta_{1}>0$ such that, for any $w^{\prime} \in U^{\prime} \backslash \bar{D}^{\prime}$ with $d\left({ }^{s} w^{\prime}, \partial D^{\prime}\right)<\delta_{1}$, the inequality $d\left(w^{\prime}, \partial D^{\prime}\right)<$ $\frac{\delta}{2}$ holds. Since $f: D \rightarrow D^{\prime}$ is proper holomorphic, there is a $\delta_{2}>0$ such that $f$ maps the set $D^{\delta_{2}}:=\left\{z \in D: d(z, \partial D)<\delta_{2}\right\}$ to the set $D^{\prime \delta_{1}}:=\left\{z^{\prime} \in D^{\prime}\right.$ : $\left.d\left(z^{\prime}, \partial D^{\prime}\right)<\delta_{1}\right\}$. We therefore suppose that our standard pair of neighborhoods $U_{1} \Subset U_{2}$ has been chosen such that $d(z, \partial D)<\delta_{2}$ for any $z \in D \cap U_{2}$. Then (b) follows.

(c) We assume that there is a sequence $\left(w^{\nu}, w^{\prime \nu}\right) \in V$ converging to a point $\left(w^{0}, w^{\prime 0}\right) \in\left(U_{1} \backslash \bar{D}\right) \times \Sigma^{\prime}$, and consider the sequence $\zeta^{\nu} \in Q_{w^{\nu}}$ such that $f\left(\zeta^{\nu}\right)=$ ${ }^{s} w^{\prime \nu}$. Since $w^{\prime \nu} \rightarrow{w^{\prime}}^{0} \in \partial D^{\prime}$, we also have ${ }^{s} w^{\prime \nu} \rightarrow^{s}{w^{\prime}}^{0}=w^{\prime 0}$ and $d\left(\zeta^{\nu}, \partial D\right) \rightarrow$ 0 . We may suppose that the sequence $\zeta^{\nu}$ converges to a point $\zeta^{0}$ and, necessarily, $\zeta^{0} \in \partial D$. The compactness assumption on $E$ implies that $\zeta^{0} \in U_{2}$, too. Hence, since $w^{\prime 0} \in \Sigma^{\prime}$, we have $\zeta^{0} \in \Sigma$ according to Theorem 6.5 , and $f\left(\zeta^{0}\right)=w^{\prime 0}$. This means that, because of Theorem 7.4, there are neighborhoods $\tilde{U}$ of $\zeta^{0}$ and $\tilde{U}^{\prime}$ of ${w^{\prime}}^{0}$ such that $f$ extends holomorphically to $D \cup \tilde{U}$ and $f \mid \tilde{U}: \tilde{U} \rightarrow \tilde{U}^{\prime}$ is proper. We put $\tilde{g}:=(f \mid \tilde{U})^{-1}$. Then we can choose $z^{\nu} \in \tilde{g}\left(w^{\prime \nu}\right)$ such that $z^{\nu} \rightarrow \zeta^{0}$. We necessarily have

$$
f\left(Q_{z^{\nu}} \cap \tilde{U}\right) \subset Q_{w^{\prime \nu}}^{\prime} \cap U_{2}^{\prime} .
$$

Therefore,

$$
Q_{z^{\nu}}=Q_{w^{\nu}}
$$

such that, by continuity also,

$$
Q_{w^{0}}=Q_{\zeta^{0}}
$$

Since, however, $\zeta^{0} \in \partial D$, one has, according to Proposition $2.2(5), A_{\zeta^{0}} \subset \partial D$. This is a contradiction to $w^{0} \notin \partial D$.

In complete symmetry to this lemma, we also have the following result:

Lemma 8.3 If $E^{\prime} \Subset U_{2}^{\prime}$, then

(a) $V^{\prime}$ is a closed complex-analytic subset of $(U \backslash \bar{D}) \times\left(U_{1}^{\prime} \backslash \bar{D}^{\prime}\right)$;

(b) there are no limit points of $V^{\prime}$ on $\partial U \times\left(U_{1}^{\prime} \backslash \bar{D}^{\prime}\right)$;

(c) if $\Sigma \subset \partial D$ denotes the set of points of holomorphic extendability of $f$, then $V^{\prime}$ does not have limit points on $\Sigma \times\left(U_{1}^{\prime} \backslash \bar{D}^{\prime}\right)$. 
Proof. Exactly the same sequence of arguments as in the proof of Lemma 8.2 may be used for this lemma. We will therefore skip repeating it here.

The decisive hypothesis in Lemmas 8.2 and 8.3 is, of course, the compactness assumption for the set $E$. A simple "geometric" criterion for this is given by the following lemma.

Lemma 8.4 Let $z^{0} \in \partial D$ be a point such that $Q_{z^{0}} \cap \bar{D}=\left\{z^{0}\right\}$. Then, for a suitable standard pair of neighborhoods $U_{1} \Subset U_{2}$ of $z^{0}$, the set $E$ as defined by (8.22) is relatively compact in $U_{2}$ (and, hence, Lemma 8.2 can be applied). In particular, this is the case if $z^{0}$ is a strictly pseudoconvex boundary point of $D$.

Proof. Observe that, according to (8.22),

$$
E \subset \bigcup_{w \in U_{1} \backslash \bar{D}} Q_{w} \cap D
$$

always holds. We now choose a neighborhood $U \Subset U_{2}$ of $z^{0}$. Since $Q_{z^{0}} \cap \bar{D}=$ $\left\{z^{0}\right\}$, we have, for a suitable constant $c>0$,

$$
d\left(Q_{z^{0}} \cap\left(U_{2} \backslash U\right), \bar{D}\right) \geq 2 c .
$$

Hence, if the neighborhood $U_{1} \Subset U$ has been chosen small enough, we also have

$$
d\left(Q_{w} \cap\left(U_{2} \backslash U\right), \bar{D}\right) \geq c
$$

for all $w \in U_{1}$, such that $\bigcup_{w \in U_{1} \backslash \bar{D}} Q_{w} \cap D \Subset U \Subset U_{2}$. This proves the lemma (because of (8.27)).

The main difficulties in applying the Segre correspondence (as defined in Definition 8.1) as the basic part of a proper holomorphic correspondence extending the map $f$ are as follows.

(1) The hypothesis of Lemma 8.4 is not satisfied at all points $z \in \partial D$;

(2) In the cases where $V$ is known to be complex-analytic, we will have to show, in addition, that the set $\hat{V}:=\bar{V} \cap\left(\left(U_{1} \backslash \bar{D}\right) \times U^{\prime}\right) \subset\left(\left(U_{1} \backslash \bar{D}\right) \times U^{\prime}\right)$ is complex-analytic and the projection $\pi: \hat{V} \rightarrow U_{1} \backslash \bar{D}$ is proper.

9. The structure of the boundary. Before we can enter into the concrete construction of the (local) proper holomorphic correspondences extending the given map $f: D \rightarrow D^{\prime}$, we have to study the boundary $\partial D$ more carefully in order to characterize a certain exceptional subset of $\partial D$, which will cause the greatest difficulties for the desired extension. For this exceptional set we will 
need to show that it is at least always pluripolar. For simplicity, we restrict ourselves in this section to the case of dimension 2 .

We will use a few facts about real-analytic and semi-analytic sets, for which we refer the reader to [23] and [12]

If $z^{0} \in \partial D$ is any point, then we can choose a neighborhood $U$ together with a real-analytic vector field $X$ on $\partial D \cap U$ with values in $T^{10} \partial D$, which vanishes nowhere on $\partial D \cap U$. Furthermore, we choose a real-analytic defining function $r$ for $D$ on $U$. We then write

$$
\mathcal{L}_{\partial D}(z):=\mathcal{L}_{r}(z ; X) .
$$

Notice, that this function is real-analytic on $U \cap \partial D$. In the following, we will use only such properties of $\mathcal{L}_{\partial D}(\cdot)$ as are independent of all the choices made here.

Since $D$ is bounded, there are always strictly pseudoconvex points on $\partial D$. We denote by $\partial D_{s}^{+}$the set of all of them. Furthermore, by $\partial D_{s}^{-}$we mean the set of points in $\partial D$, where the Levi form $\mathcal{L}_{\partial D}$ is strictly negative. Because of the main results of [17] and [3], we may, of course, always assume in this article without mentioning that $\partial D_{s}^{-} \neq \varnothing$.

We call the set

$$
\partial D^{+}:=\frac{\circ}{\partial D_{s}^{+}}
$$

the pseudoconvex region of $\partial D$ (the interior is taken in the relative topology on $\partial D)$, and the set

$$
\partial D^{-}:=\frac{\circ}{\partial D_{s}^{-}}
$$

the pseudoconcave region of $\partial D$. It is well known that $\partial D^{-} \subset \hat{D}$, where $\hat{D}$ denotes as always the envelope of holomorphy of $D$.

In some sense the most interesting part of $\partial D$ is the remaining rest.

Definition 9.1 The set $M:=\partial D \backslash\left(\partial D^{+} \cup \partial D^{-}\right)$is called the border in $\partial D$.

\section{Remarks.}

(a) On $\partial D$, the border $M$ separates the pseudoconcave from the pseudoconvex region.

(b) It should not be forgotten that, besides $\partial D^{-}$, other parts of $\partial D$ may also lie in $\hat{D}$. (In fact, even relatively open subsets of $\partial D_{s}^{+}$might do this.) For the purpose of this article it is even crucial to know that, in some sense (see below), most of $M$ is contained in $\hat{D}$, a fact that was first proved in $[15]$. 
Next we introduce

$$
T:=\left\{z \in \partial D: \mathcal{L}_{\partial D}(z)=0\right\} .
$$

Notice that $T$ is a compact real-analytic subset of $\partial D$ of dimension $\leq 2$ and, obviously, $M \subset T$. In fact, it is easy to see that $M$ is always a closed semianalytic set.

We put $T^{+}:=T \cap \partial D^{+}$and call it the set of weakly pseudoconvex points on $\partial D$, and $T^{-}:=T \cap \partial D^{-}$and call it the set of weakly pseudoconcave points of $\partial D$. Then, one has the following result:

Lemma 9.2 The set $T$ can be stratified in the following way by semianalytic sets

$$
T=T_{2} \cup T_{1} \cup T_{0}
$$

where $T_{k}$ is a locally finite set of smooth real-analytic submanifolds of dimension $k$ for $k=0,1,2$. The set $T_{1} \cup T_{0}$ is real-analytic (of dimension $\leq 1$ ).

Our next goal is to refine this stratification in a way needed for our later purposes. For this, we observe that the set

$$
T^{h}:=\left\{z \in T_{2}: \operatorname{dim} T_{z}^{10}\left(T_{2}\right)=1\right\}
$$

is semi-analytic of dimension $\leq 1$ in $T_{2}$ since, otherwise, there would be a piece of a complex-analytic curve contained in $\partial D$. This is, however, impossible according to [14]. Notice that $T_{2} \backslash T^{h}$ is maximally totally real.

Furthermore, if we denote by $o_{\mathcal{L}}(z)$ the order of vanishing of the Levi form $\mathcal{L}_{\partial D}$ at the point $z \in \partial D$, then the set

$$
T^{d}:=\left\{z \in T: \varliminf_{T \ni \zeta \rightarrow z} o_{\mathcal{L}}(\zeta)<o_{\mathcal{L}}(z)\right\}
$$

is real-analytic of dimension $\leq 1$.

From this construction, the next result follows.

Lemma 9.3 The set $T_{1} \cup T_{0} \cup T^{h} \cup T^{d}$ can be stratified in the form $\hat{T}_{1} \cup \hat{T}_{0}$, where $\hat{T}_{1}$ is a locally finite family of smooth curves and $\hat{T}_{0}$ a locally finite family of points. In particular, the set $\hat{T}_{1} \cup \hat{T}_{0}$ is pluripolar. The set $\hat{T}_{2}:=T_{2} \backslash\left(\hat{T}_{1} \cup \hat{T}_{0}\right)$ is a locally finite family of maximally totally real submanifolds.

In what follows, in order to keep our notation as simple as possible, we make the following chance.

Change of notation: From now on we write $T_{k}$ instead of $\hat{T}_{k}$ for $k=0,1,2$, and we put $T_{k}^{+}:=T_{k} \cap \partial D^{+}$.

The following result was shown in [15].

Theorem 9.4 The set $M_{e}:=M \cap\left(T_{1} \cup T_{0}\right)$ is semi-analytic and $\hat{m}:=$ $M \backslash M_{e} \subset \hat{D}$. 
10. Extension across a dense subset of the boundary. As a starting point for extending the given map $f: D \rightarrow D^{\prime}$ as a proper holomorphic correspondence, we need to know that it extends as a proper holomorphic map across an open dense subset of $\partial D$.

Theorem 10.1 Let $D, D^{\prime} \Subset \mathbb{C}^{2}$ be domains with smooth real-analytic boundaries and $f: D \rightarrow D^{\prime}$ a proper holomorphic mapping. Then there is a dense, open subset $\Sigma \subset \partial D$, such that $f$ extends holomorphically to a neighborhood of $D \cup \Sigma$. The set $\Sigma$ can even be chosen in such a way that this extension is locally biholomorphic at all points $a \in \Sigma$.

Since $\partial D_{s}^{-} \subset \hat{D}, f$ extends as a proper holomorphic map across $\partial D_{s}^{-}$. This extension is locally biholomorphic almost everywhere in $\partial D_{s}^{-}$. Therefore, we only have to consider $\partial D_{s}^{+}$. Suppose that there is a point $a \in \partial D_{s}^{+}$with a (relatively) open neighborhood $S \subset \partial D_{s}^{+}$of $a$, such that $f$ does not extend holomorphically to a neighborhood of any point $z \in S$. In this situation, we first show this fact.

Claim 1: For all $z \in S$, the cluster set $\operatorname{cl}_{f}(z) \subset M_{e}^{\prime}$, where $M_{e}^{\prime}$ is the exceptional set of Theorem 9.4.

Proof. Namely, if $\mathrm{cl}_{f}(z)$ contained a point $z^{\prime} \in \hat{D}$, then $z \in \hat{D}$ would also, according to Lemma 3.1. If, on the other hand, $\mathrm{cl}_{f}(z)$ contains a point $z^{\prime} \in \partial{D^{\prime}}^{+}$, i.e., the pseudoconvex region of $\partial D$, then, because of a theorem of F. Berteloot [11], $f$ extends continuously to $\partial D \cup V$ for a certain neighborhood $V$ of $z$. The image of $S \cap V$ under this extension necessarily lies in $\partial{D^{\prime}}^{+}$and, hence, the extension is even locally biholomorphic at all points $z \in S \cap V$. This proves Claim 1.

We now use the information of Theorem 9.4, which says that $M_{e}^{\prime}$ is pluripolar. Therefore, there exists a function $\varphi \neq-\infty$ plurisubharmonic on $\mathbb{C}^{2}$ and such that $\varphi \mid M_{e}^{\prime} \equiv-\infty$. The function $\psi:=\varphi \circ f$ is plurisubharmonic on $D$ and if $D \ni z \rightarrow z^{0} \in S$, then $\psi \rightarrow-\infty$. Since, however, $S \subset \partial D$ is relatively open, it follows that $\psi \equiv-\infty$, such that $\varphi$ would also be $\equiv-\infty$ on $D$-a contradiction.

Before we come to a statement symmetric to Theorem 10.1, we introduce the following.

Definition 10.2 Let $a^{\prime} \in \partial D^{\prime}$ and $U^{\prime}$ a (small) open neighborhood of $a^{\prime}$. If $g: D^{\prime} \cap U^{\prime} \rightarrow U$ is a proper holomorphic correspondence, then we call the number $\# g\left(w^{\prime}\right)$ a degree of $g$ for generic $w^{\prime} \in U^{\prime}$.

Analogously to Theorem 10.1, the following also holds. 
Theorem 10.3 In the situation of Theorem 10.1. there exists a (relatively) open dense subset $\Sigma^{\prime} \subset \partial D^{\prime}$, such that the global inverse $g=f^{-1}$ extends as a proper holomorphic correspondence to a neighborhood of any point $a^{\prime} \in \Sigma^{\prime}$.

Proof. As in the previous proof, it suffices to show that $g$ extends as a proper holomorphic correspondence to a neighborhood of any point on an open dense subset of $\partial D_{s}^{\prime+}$. Suppose again that this is not true. Then there is a point $a^{\prime} \in \partial{D_{s}^{\prime}}_{s}^{+}$and a (relatively) open neighborhood $S^{\prime} \subset \partial{D_{s}^{\prime}}^{+}$of $a^{\prime}$ such that, for any $z^{\prime} \in S^{\prime}$, the global inverse $g$ does not extend as a proper holomorphic correspondence to a neighborhood of $z^{\prime}$.

We show at first:

Claim 1: Suppose that, for a point ${z^{\prime}}^{0} \in S$, there is a point $z^{0} \in$ $\operatorname{cl}_{g}\left({z^{\prime}}^{0}\right) \cap \hat{D}$ or a point $z^{0} \in \operatorname{cl}_{g}\left({z^{\prime}}^{0}\right) \cap \partial D^{+}$. Then $g$ extends as a proper holomorphic correspondence to a neighborhood of the pair $\left(z^{0}, z^{\prime 0}\right)$ (in the sense of Definition 6.2).

Namely, notice that ${z^{\prime}}^{0} \in \mathrm{cl}_{f}\left(z^{0}\right)$. If $z^{0} \in \hat{D}, f$ extends holomorphically to a neighborhood of $z^{0}$. Hence Theorem 6.3 does the job. If $z^{0} \in \operatorname{cl}_{g}\left(z^{\prime 0}\right) \cap \partial D^{+}$, then $f$ extends continuously to a neighborhood of $z^{0}$ (again according to the result by F. Berteloot). Applying a theorem by S. Bell and D. Catlin [9] gives the $\mathcal{C}^{\infty}$-extendability of $f$ to a neighborhood of $z^{0}$ and, finally, it follows from [17] that $f$ extends as a proper holomorphic map to a neighborhood of $z^{0}$. Hence, again because of Theorem 6.3, $g$ extends as a proper holomorphic correspondence to a neighborhood of the pair $\left(z^{0}, z^{\prime 0}\right)$. This proves Claim 1 .

Notice that, whenever $g$ extends as a proper holomorphic correspondence to a neighborhood of the pair $\left(z^{0}, z^{\prime 0}\right)$, then, for a suitable neighborhood $U^{\prime}$ of ${z^{\prime}}^{0}$, the restriction $\Gamma_{g}\left(U^{\prime}\right):=\Gamma_{g} \mid\left\{D \times\left(U^{\prime} \cap D^{\prime}\right)\right\}$ can be represented in the form

$$
\Gamma_{g}\left(U^{\prime}\right)=\Gamma_{1}\left(U^{\prime}\right) \cup \Gamma_{2}\left(U^{\prime}\right),
$$

where $\Gamma_{1}\left(U^{\prime}\right)$ and $\Gamma_{2}\left(U^{\prime}\right)$ are closed analytic subsets of $D \times\left(U^{\prime} \cap D^{\prime}\right)$ with proper projections to $U \cap D^{\prime}$, and where the set $\Gamma_{1}\left(U^{\prime}\right)$ corresponds to the points from $\operatorname{cl}_{g}\left({z^{\prime}}^{0}\right) \cap\left(\partial D^{+} \cup \hat{D}\right)$. It extends (after possibly shrinking $U^{\prime}$ ) to a proper holomorphic correspondence over $U^{\prime}$. The set $\Gamma_{2}\left(U^{\prime}\right)$ is the "rest" of the graph $\Gamma_{g}\left(U^{\prime}\right)$ and corresponds to the points from $\operatorname{cl}_{g}\left({z^{\prime}}^{0}\right) \cap M_{\varepsilon}$. More precisely, it is the graph of a proper holomorphic correspondence $g^{2}: U^{\prime} \cap D^{\prime} \rightarrow D$ (interpreted as a multi-valued map) with the property $\operatorname{cl}_{g^{2}}\left({z^{\prime}}^{0}\right) \subset M_{\varepsilon}$. If $\Gamma_{1}\left(U^{\prime}\right) \neq \varnothing$, then the degree $m_{2}$ of $g_{2}$ satisfies $m_{2}<m$, where $m$ is the sheet number of $f$.

Suppose now that there is (still) a point $z^{\prime 1} \in S^{\prime} \cap U^{\prime}$ such that $\operatorname{cl}_{g^{2}}\left(z^{\prime 1}\right) \nsubseteq$ $M_{e}$. Then we can repeat the procedure above and split from $\Gamma_{g^{2}}\left(U^{\prime \prime}\right), U^{\prime \prime} \in$ $z^{\prime 1}$, another "remainder" with an even lower degree. After a finite number of 
steps we get a point $a^{\prime} \in S^{\prime}$ with a neighborhood $\tilde{U}$ and a proper holomorphic correspondence $\tilde{g}: D^{\prime} \cap \tilde{U} \rightarrow D$ such that $f \circ \tilde{g}=i d$ and $\operatorname{cl}_{\tilde{g}}(S \cap \tilde{U}) \subset M_{e}$. Now, let $\varphi$ again be a plurisubharmonic function on $\mathbb{C}^{2}$, such that $\varphi \mid M_{e} \equiv-\infty$. The function

$$
\psi\left(z^{\prime}\right):=\max _{z \in \tilde{g}\left(z^{\prime}\right)} \varphi(z)
$$

is now plurisubharmonic in $U^{\prime} \cap D^{\prime}$ and $\psi\left(z^{\prime}\right) \rightarrow-\infty$ if $z^{\prime} \rightarrow z^{\prime 0} \in S \cap \tilde{U}$. Therefore, $\psi \equiv-\infty$-a contradiction.

This contradiction shows that there is a (relatively) open subset $\hat{U} \Subset S^{\prime}$ such that $\operatorname{cl}_{g}\left(z^{\prime}\right) \subset \partial D^{+} \cup \hat{D}$ for all $z^{\prime} \in \hat{U}$. Claim 1 therefore says that the global inverse $g$ extends as a proper holomorphic correspondence to a neighborhood of any point $z^{\prime} \in \hat{U}$. This is a contradiction to our assumption about the set $S^{\prime}$, thus proving the theorem.

Now that we know that the given map $f: D \rightarrow D^{\prime}$ extends biholomorphically across an open dense subset of $\partial D$, we can make the following useful change in the definition of the variety $V$ from Definition 8.1. Namely, the variety $V$ as defined at first might contain useless components. Therefore, we now replace it by the following part of it:

Definition 10.4 Suppose the situation is as in part (1) of Definition 8.1. Choose any point $a \in \partial D \cap U_{1}$ of biholomorphic extendability of $f$. Suppose, furthermore, that $E \Subset U_{2}$. Then we denote by $V$ the irreducible component in $\left(U_{1} \backslash \bar{D}\right) \times\left(U^{\prime} \backslash \bar{D}^{\prime}\right)$ of the variety given by (8.21), which contains the graph of the extended map $f$ near $a$.

\section{Remarks.}

(a) According to Lemma 8.2, $\mathrm{V}$ is indeed a closed complex-analytic variety.

(b) As it will turn out later, the variety $V$ defined here does not depend on the choice of the point $a$.

11. Extension across strictly pseudoconvex points. The tools for extending the given map $f: D \rightarrow D^{\prime}$ as a proper holomorphic correspondence, which were given by Lemmas 8.2 and 8.3 together with Definition 10.4, can most easily be applied near strictly pseudoconvex boundary points. We show:

Lemma 11.1 Let $z^{0} \in \partial D_{s}^{+}$. Then $z^{0}$ is a point of local biholomorphic extendability of $f$.

Proof. Because of Theorem 7.4 we only have to show, that $f$ extends as a proper holomorphic correspondence to a neighborhood of $z^{0}$. For this, we 
choose neighborhoods $U_{1} \subset \subset U_{2}$ of $z^{0}$ and $U^{\prime}$ of $\partial D^{\prime}$ as in the definition of the Segre correspondence (Definition 8.1). According to Lemmas 8.4 and 8.2 the Segre correspondence $V$ is, then, a closed complex-analytic subvariety of the set $\left(U_{1} \backslash \bar{D}\right) \times\left(U^{\prime} \backslash \bar{D}^{\prime}\right)$. We, therefore, mean by $V$ from now on the part given by Definition 10.4. The main work, we still have to do, is to show, that $V$ is contained in a closed complex-analytic subset $\hat{V} \subset\left(U_{1} \backslash \bar{D}\right) \times U^{\prime}$ such that the projection $\pi: \widehat{V} \rightarrow U_{1} \backslash \bar{D}$ is proper. For this, we observe at first the following:

Claim 1: $V$ does not have any limit point on $\left(U_{1} \backslash \bar{D}\right) \times\left(\partial U^{\prime} \cup\left(\partial D^{\prime} \backslash\right.\right.$ $\left.\left.M_{e}^{\prime}\right)\right)$.

Namely, according to part (b) of Lemma 8.2, the set $V$ does not have limit points on $\left(U_{1} \backslash \bar{D}\right) \times \partial U^{\prime}$, and according to part (c) of the same lemma, $V$ does not have any limit point on the set

$$
\left(U_{1} \backslash \bar{D}\right) \times\left(\partial D^{\prime} \cap \hat{D}^{\prime}\right) .
$$

Let us, therefore, suppose that $V$ has a limit point $\left(w^{0}, w^{\prime 0}\right) \in\left(U_{1} \backslash \bar{D}\right) \times \partial D^{\prime+}$. Then there is a sequence $\left(w^{k}, w^{\prime k}\right) \in V$ with $\left(w^{k}, w^{\prime k}\right) \rightarrow\left(w^{0}, w^{\prime 0}\right)$. We choose $z^{k} \in Q_{w^{k}}$ such that $f\left(z^{k}\right)={ }^{s} w^{\prime k}$. Since ${ }^{s} w^{\prime k} \rightarrow w^{\prime 0} \in \partial D^{\prime+}$, given (8.21) we may suppose (after taking a suitable subsequence) that the sequence of the $z^{k}$ converges to a point $\tilde{z}$ which, necessarily, lies in $\partial D \cap \overline{U_{2}}$. However, because of Lemma 8.4, $\tilde{z}$ cannot lie on $\partial U_{2}$. Hence, $\tilde{z} \in \partial D_{s}^{+} \cap U_{2}$. Therefore, we have shown that the set $\operatorname{cl}_{\mathrm{f}}(\tilde{z})$ contains the point ${w^{\prime}}^{0} \in \partial D^{\prime+}$, where $\partial D$ is, of course, of finite type. The result of $\mathrm{F}$. Berteloot from [11] implies that $f$ extends in a Hölder continuous way to $\bar{D} \cap U$ for a certain neighborhood $U$ of $\tilde{z}$. Hence, the theorem of S. Bell and Catlin from [9] tells us that $f$ extends even in a $\mathcal{C}^{\infty}$ way to $\bar{D} \cap U$, from which it follows that $f$ is locally biholomorphic on $U$ according to [17] and [3]. By continuity of the Segre varieties it also follows that $z Q_{w^{0}}={ }_{z} Q_{\tilde{z}}$. This, however, contradicts Proposition 2.2.

The possibility still unaccounted for is that there are limit points of $V$ on $\left(U_{1} \backslash \bar{D}\right) \times \partial D^{\prime}$ lying on the set $\left(U_{1} \backslash \bar{D}\right) \times M_{e}^{\prime}$. Then $V$ is even closed and complex-analytic in $\left(\left(U_{1} \backslash \bar{D}\right) \times U^{\prime}\right) \backslash E^{\prime}$, where we use the notation $E^{\prime}:=$ $\left(U_{1} \backslash \bar{D}\right) \times M_{e}^{\prime} \subset\left(U_{1} \backslash \bar{D}\right) \times U^{\prime}$. But, according to Lemma 9.3 and Theorem 9.4, we know that $E^{\prime}$ is pluripolar. So, there is a plurisubharmonic function $\varphi \neq-\infty$ on $\left(U_{1} \backslash \bar{D}\right) \times U^{\prime}$, such that $E^{\prime} \subset\left\{\left(z, z^{\prime}\right) \in\left(U_{1} \backslash \bar{D}\right) \times U: \varphi\left(z, z^{\prime}\right)=-\infty\right\}$. We put $\tilde{E}^{\prime}:=\left\{\left(z, z^{\prime}\right) \in\left(U_{1} \backslash \bar{D}\right) \times U^{\prime}: \varphi\left(z, z^{\prime}\right)=-\infty\right\}$ and $\tilde{V}:=V \cap\left[\left(\left(U_{1} \backslash \bar{D}\right) \times U^{\prime}\right) \backslash \tilde{E}^{\prime}\right]$. Then $\tilde{V}$ is a closed complex-analytic subset of $\left(\left(U_{1} \backslash \bar{D}\right) \times U^{\prime}\right) \backslash \tilde{E}^{\prime}$; we have $\tilde{V} \neq \varnothing$ and $\operatorname{dim} \tilde{V} \equiv 2$. However, there are points $z \in U_{1} \cap \partial D$ such that the map $f$ extends biholomorphically to a neighborhood $\tilde{U}$ of $z$. Therefore, on $(\tilde{U} \backslash \bar{D}) \times U^{\prime}$, the Segre correspondence $V$ is just the graph of a holomorphic mapping. Indeed, let $\left(w, w^{\prime}\right) \in V$ with $w \in \tilde{U} \backslash \bar{D}$. Since $\tilde{U}$ is small, then $Q_{w} \cap D$ and $f\left(Q_{w} \cap D\right)$ are small and close to $z$ and $f(z)$, respectively. Therefore, ${ }^{s} w^{\prime} \in f\left(Q_{w} \cap D\right)$ is 
also close to $f(z)$. Since $f(z) \in \partial{D_{s}^{\prime}}^{+}$, the mapping $\lambda^{\prime}$ is locally one-to-one near $f(z)$, and we conclude that $w^{\prime}$ is uniquely defined, i.e., $w^{\prime}=f(w)$.

This shows that we have the situation of the so-called Bishop's Lemma (see [13], p. 240), which tells us that $\hat{V}:=\overline{\tilde{V}}$ is a closed complex-analytic subset of $\left(U_{1} \backslash \bar{D}\right) \times U^{\prime}$ of pure dimension 2 without limit points on $\left(U_{1} \backslash \bar{D}\right) \times \partial U^{\prime}$. Hence, the projection $\pi: \hat{V} \rightarrow U_{1} \backslash \bar{D}$ is proper. We also have $\hat{V} \cap\left(\left(U_{1} \backslash \bar{D}\right) \times\left(U^{\prime} \backslash \bar{D}^{\prime}\right)=V\right.$.

The map $\pi: \hat{V} \rightarrow U_{1} \backslash \bar{D}$ is proper holomorphic, hence a finite-branched, covering, the sheet number of which we denote by $m$. From this, it is easy to obtain an extension of $f$ as a proper holomorphic correspondence to a neighborhood of $z^{0}$. Namely, it follows that there are two pseudopolynomials of the form

$$
P_{k}\left(z ; z_{k}^{\prime}\right)=z_{k}^{\prime m}+a_{k 1}(z) z^{\prime m-1}+\cdots+a_{k m}(z)=0
$$

with holomorphic coefficients $a_{k j}(\cdot) \in \mathcal{O}\left(U_{1} \backslash \bar{D}\right)$, such that

$$
\hat{V} \subset \tilde{F}_{1}:=\left\{\left(z, z^{\prime}\right) \in\left(U_{1} \backslash \bar{D}\right) \times U^{\prime}: P_{k}\left(z ; z_{k}\right)=0\right\}
$$

If $U$ is a small enough neighborhood of $z^{0}$, then all coefficients $a_{k j}$ extend to holomorphic functions on $U$ such that we get

$$
\hat{V} \subset \tilde{F}:=\left\{.\left(z, z^{\prime}\right) \in U \times U^{\prime}: P_{k}\left(z ; z_{k}^{\prime}\right)=0, k=1,2\right\},
$$

where $\tilde{F}$ is a closed complex-analytic subset of $U \times U^{\prime}$ containing $\Gamma_{f} \mid D \cap U$. The irreducible component $F$ of $\tilde{F}$ containing $\Gamma_{f} \mid D \cap U$ gives us the desired proper holomorphic correspondence extending $f$ to $U$.

Lemma 11.1 has the following analogue for the extendability of the global inverse $g=f^{-1}$ :

Lemma 11.2 Let ${z^{\prime}}^{0} \in \partial D_{s}^{\prime+}$. Then ${z^{\prime}}^{0} \in \Sigma^{\prime}$, which means that $g$ extends as a proper holomorphic correspondence to a neighborhood of ${z^{\prime}}^{0}$ (in the sense of Definition 6.1).

Proof. The proof is the same as in Lemma 1.

12. Final steps for proving Theorem 1.2. It remains to extend $f$ holomorphically beyond the "rest" of $\partial D$, namely, the set $T^{+} \cup M_{\varepsilon}$. We will do this in a step-by-step approach by constructing local extensions as proper holomorphic correspondences and then applying Theorem 7.4. At the same time, we will obtain (and, in fact, need) an extension of $g=f^{-1}$ as a proper holomorphic correspondence to $T^{\prime+} \cup M_{\varepsilon}^{\prime}$.

We first show another fact about the Segre varieties, which will be very useful: 
Lemma 12.1 For any point $z^{0} \in T_{2}^{+}$there exist arbitrarily small standard neighborhoods $U_{1} \Subset U_{2}$ of $z^{0}$ such that

$$
\left(Q_{z^{0}} \cap \partial D\right) \backslash\left\{z^{0}\right\} \subset \partial D_{s}^{+} .
$$

Proof. After an appropriate local holomorphic change of coordinates, we may assume that $z^{0}=0$ and that the totally real submanifold $T_{2}^{+}$of dimension 2 is just the real plane $i \mathbb{R}^{2}$ near $z^{0}$. Hence, a local real-analytic defining function $r$ of $D$ near 0 may be chosen in the form

$$
r(z)=2 x_{2}+\left(2 x_{1}\right)^{2 m} a\left(z_{1}, y_{2}\right)
$$

with $m>1$ and a real-analytic function $a\left(z, y_{2}\right)>0$ in a neighborhood of 0 . The complexification of $r$ has the form

$$
r(z, w)=z_{2}+\overline{w_{2}}+\left(z_{1}+\overline{w_{1}}\right)^{2 m} \tilde{a}(z, w)
$$

with $\tilde{a}(0,0)>0$. Therefore, $Q_{0}$ is locally given by the equation

$$
z_{2}+z_{1}^{2 m} \tilde{a}(z, 0)=0 .
$$

Its restriction to $T_{2}^{+}$is

$$
i y_{2}+(-1)^{m} y_{1}^{2 m} \tilde{a}(i y, 0)=0 .
$$

Since $\operatorname{Re} \tilde{a}(i y, 0)>0$, the Segre variety $Q_{0}$ intersects $T_{2}^{+}$only at the origin.

In the next lemma we consider another situation in which the set $V$, as defined by (8.21), turns out to be useful for the construction of a proper holomorphic correspondence extending $f$.

Lemma 12.2 Let $z^{0} \in \partial D, U^{\prime}$ a suitable neighborhood of $\partial D^{\prime}, U_{1} \Subset U_{2}$ a suitable standard pair of neighborhoods of $z^{0}$ and $V \subset\left(U_{1} \backslash \bar{D}\right) \times\left(U^{\prime} \backslash \bar{D}^{\prime}\right)$ as given by (8.21). If $\left(\left(Q_{z^{0}} \cap \partial D\right) \backslash\left\{z^{0}\right\}\right) \subset \Sigma$, the set of points of holomorphic extendability of $f$, then $V$ is a closed complex-analytic subset of $\left(U_{1} \backslash \bar{D}\right) \times\left(U^{\prime} \backslash \bar{D}^{\prime}\right)$ without any limit points on $\left(U_{1} \backslash \bar{D}\right) \times\left(\partial U^{\prime} \cup \Sigma^{\prime}\right)$.

Proof. Shrinking $U_{2}$, if necessary, we may assume that $Q_{z^{0}} \cap \partial D \cap \partial U_{2} \subset \Sigma$ and that there exists a radius $\tau_{1}>0$ such that, for all $w \in Q_{z^{0}} \cap \partial U_{2} \cap \bar{D}$, the map $f$ extends holomorphically to the ball of radius $\tau_{1}$ centered at $w$. Let $R^{\prime}\left(z^{0}\right)$ be the set of all points $w^{\prime} \in U^{\prime} \backslash \bar{D}^{\prime}$ for which

$$
f\left(Q_{z^{0}} \cap D\right) \supset{ }_{s_{w^{\prime}}} Q_{w^{\prime}}^{\prime} .
$$


Then $R^{\prime}\left(z^{0}\right)$ is discrete in $U^{\prime} \backslash \bar{D}^{\prime}$, because the map $\lambda^{\prime}$ is finite. Therefore, the set

$$
R\left(z^{0}\right):=\left\{w \in Q_{z^{0}} \cap D \cap \overline{U_{2}}: f(w)={ }^{s} w^{\prime} \quad \text { for some } w^{\prime} \in R^{\prime}\left(z^{0}\right)\right\}
$$

also is discrete in $D$. Shrinking $U_{2}$ and $U_{1}$ again, we may assume that

$$
R\left(z^{0}\right) \cap \partial U_{2}=\varnothing,
$$

and, moreover, there exists a radius $\tau_{2}>0$ such that, for all $w \in U_{1} \backslash \bar{D}$, the set $R(w)$ (defined as in (12.40)) does not intersect the neighborhood of width $\tau_{2} / 2$ of the set $Q_{w} \cap D \cap \partial U_{2}$ (of course, such a neighborhood of the empty set is taken to be empty). Hence, the hypothesis $E \Subset U_{2}$ of Lemma 8.2 is satisfied, such that $V$ is a closed complex-analytic subset of $\left(U_{1} \backslash \bar{D}\right) \times\left(U^{\prime} \backslash \bar{D}^{\prime}\right)$ without limit points on $(U \backslash \bar{D}) \times\left(\partial U^{\prime} \cup \Sigma^{\prime}\right)$.

In total symmetry to Lemma 12.2 , we also have the following result.

Lemma 12.3 Let ${z^{\prime}}^{0} \in \partial D^{\prime}, U$ a suitable neighborhood of $\partial D, U_{1}^{\prime} \Subset U_{2}^{\prime}$ a suitable standard pair of neighborhoods of ${z^{\prime}}^{0}$ and $V^{\prime} \subset(U \backslash \bar{D}) \times\left(U_{1}^{\prime} \backslash \bar{D}^{\prime}\right)$ as given by (8.23). If $\left(\left(Q_{z^{\prime 0}}^{\prime} \cap \partial D^{\prime}\right) \backslash\left\{z^{\prime 0}\right\}\right) \subset \Sigma^{\prime}$, the set of points of extendability of the global inverse $g=f^{-1}$, then $V^{\prime}$ is a closed complex-analytic subset of $(U \backslash \bar{D}) \times\left(U_{1}^{\prime} \backslash \bar{D}^{\prime}\right)$ without limit points on $(\partial U \cup \Sigma) \times\left(U_{1}^{\prime} \backslash \bar{D}^{\prime}\right)$.

Proof. The arguments are totally analogous to the proof of Lemma 12.2.

Our essential additional goal in all situations, where $V$ is known to be a closed complex-analytic subset of $\left(U_{1} \backslash \bar{D}\right) \times\left(U^{\prime} \backslash \bar{D}^{\prime}\right)$, is to show that the projection $\pi$ : $V \rightarrow\left(U_{1} \backslash \bar{D}\right)$ is proper. Then it can immediately be used to obtain an extending proper holomorphic correspondence and, hence, holomorphic extendability of $f$. We consider at first the following case.

Lemma 12.4 One has $T_{2}^{+} \subset \Sigma$.

Proof. Let $z^{0} \in T_{2}^{+}$and make all choices as in Lemma 12.1. Then we have $\left(Q_{z^{0}} \cap \partial D\right) \backslash\left\{z^{0}\right\} \subset \partial D_{s}^{+}$. By Theorem 11.1, we get

$$
\left(Q_{z^{0}} \cap \partial D\right) \backslash\left\{z^{0}\right\} \subset \Sigma
$$

Notice that in the case $\operatorname{cl}_{f}\left(z^{0}\right) \cap \Sigma^{\prime} \neq \varnothing$, because of Theorem 6.5 together with Theorem 7.4, we have $z^{0} \in \Sigma$. Because of the results of F. Berteloot [11], S. Bell and D. Catlin [9] and [17], this means, in particular, that we are done if 
$\operatorname{cl}_{f}\left(z^{0}\right) \cap \partial{D^{\prime}}^{+} \neq \varnothing$. Since, furthermore, $\partial{D^{\prime}}^{-} \subset \Sigma^{\prime}$, we only need to consider the case where

$$
\operatorname{cl}_{f}\left(z^{0}\right) \subset\left(T_{1}^{\prime} \cup T_{0}^{\prime}\right) \backslash \partial D^{\prime-} .
$$

However, according to Lemma 9.3, the set $T_{1}^{\prime} \cup T_{0}^{\prime}$ is pluripolar, which enables us to use Bishop's Lemma if we know that the following holds:

Claim: For a suitable choice of $U_{1}$ there is a non-empty open set $\tilde{U} \subset U_{1} \backslash \bar{D}$ such that $V \cap\left(\tilde{U} \times\left(U^{\prime} \backslash \bar{D}^{\prime}\right)\right)$ is a closed complex-analytic subset of $\tilde{U} \times U^{\prime}$.

In order to deduce this claim, we have to choose $U_{1} \Subset U_{2}$ at first very carefully. Namely, $Q_{z^{0}}$ is a complex manifold of dimension 1 and $\lambda$ is an open map. Therefore, for any (small) $\delta>0$ and $U_{0}:=\left\{z:\left\|z-z^{0}\right\|<\delta\right\}$, the set

$$
W:=\bigcup_{w \in U_{0}} Q_{w}
$$

is an arbitrarily small neighborhood of $Q_{z^{0}}$. Let us, for the moment, denote by $T_{2 \delta}^{+}$the neighborhood of $T_{2}^{+}$of width $\frac{\delta}{2}$. We then choose $U_{1}$ such that $U_{1} \cap T_{2 \delta}^{+} \subset$ $U_{0}$, but there also exists a non-empty open set $\tilde{U} \Subset U_{1} \backslash(\bar{W} \cup \bar{D})$. It then necessarily follows that, for $z \in \tilde{U}, Q_{z} \cap U_{0}=\varnothing$. Hence, for all $\tilde{z} \in U$, we have

$$
Q_{z} \cap \partial D \subset \partial D_{s}^{+} \subset \Sigma .
$$

This implies that $f$ is well defined on $Q_{z} \cap \partial D$ and

$$
f\left(Q_{z} \cap \partial D\right) \subset \partial D_{s}^{\prime+} \subset \Sigma^{\prime} .
$$

We now conclude as follows.

The set $V \cap\left\{\tilde{U} \times\left(U^{\prime} \backslash \bar{D}^{\prime}\right)\right\}$ has no limit points on $\tilde{U} \times \partial D^{\prime}$. Otherwise, for a point $\left(z^{0}, z^{\prime 0}\right) \in \bar{V} \cap\left(\tilde{U} \times \partial D^{\prime}\right)$ we would get, as in Part (3) of the proof of Lemma 8.2, that ${z^{\prime}}^{0} \in f\left(Q_{z^{0}}\right) \cap \partial D^{\prime} \subset \Sigma^{\prime}$. This is a contradiction to Lemma 12.2, thus proving the Claim.

Now Bishop's Lemma implies that $\bar{V}$ is an analytic set in $\left(U_{1} \backslash \bar{D}\right) \times U^{\prime}$. Since it has no limit points on $\left(U_{1} \backslash \bar{D}\right) \times \partial U^{\prime}$, the map $f$ can be extended as a proper holomorphic correspondence to a neighborhood of the point $z^{0}$, exactly as in the second half of the proof of Lemma 11.1. This finishes the proof of Lemma 12.4.

Again we also have the following symmetric statement by the symmetric arguments using the fact that we have already obtained all results symmetric to those used in the proof of Lemma 12.4.

Lemma 12.5 One has $T_{2}^{+} \subset \Sigma^{\prime}$. 
In order to finish the proof of Theorem 1.2 completely, we still have to consider the points of $\left(T \backslash T_{2}\right) \backslash \partial D^{-}$. For these points we deal at first with the following simple case:

Lemma 12.6 Let $z^{0} \in\left(T \backslash T_{2}\right) \backslash \partial D^{-}$and suppose, in addition, that

$$
\left(Q_{z^{0}} \cap \partial D\right) \backslash\left\{z^{0}\right\} \subset \Sigma .
$$

Then $z^{0} \in \Sigma$.

The symmetric statement will no longer be needed.

Proof. Since we already know that $\partial D^{\prime} \backslash\left(T_{1}^{\prime} \cup T_{0}^{\prime}\right) \subset \Sigma^{\prime}$, we get from Lemma 12.2 that the set $V$, as considered there, does not have any limit points on $\left(U_{1} \backslash \bar{D}\right) \times\left\{\partial D^{\prime} \backslash\left(T_{1}^{\prime} \cup T_{0}^{\prime}\right)\right\}$, nor on $\left(U_{1} \backslash \bar{D}\right) \times \partial U^{\prime}$. The same as above, this allows us again to apply Bishop's Lemma in order to show that, in fact, $\bar{V}$ is an analytic set in $\left(U_{1} \backslash \bar{D}\right) \times U^{\prime}$. As we already know, this implies $z^{0} \in \Sigma$.

What remains is the situation where, for a certain point $z^{0} \in\left(T \backslash T_{2}\right) \backslash \partial D^{-}$,

$$
\left(Q_{z^{0}} \cap\left(T_{1} \cup T_{0}\right)\right) \backslash\left\{z^{0}\right\} \neq \varnothing .
$$

If in this case $Q_{z^{0}} \cap\left(T_{1} \cup T_{0}\right)$ is discrete, we can just shrink $U_{1}$ and $U_{2}$ and are in the situation of Lemma 12.6. Therefore, it now suffices to show the following.

Lemma 12.7 Let $z^{0} \in\left(T \backslash T_{2}\right) \backslash \partial D^{-}$, and suppose that $Q_{z^{0}} \cap\left(T_{1} \cup T_{0}\right)$ contains a real-analytic set of dimension 1 near $z^{0}$. Then $z^{0} \in \Sigma$.

Again, the symmetric statement is no longer needed.

Proof. If $U_{1}$ is suitably chosen small enough, then any of the finitely many smooth curves contained in the set $\left(T_{1} \cup T_{0}\right) \cap U_{1}$ has a complexification $\hat{T}$. Then the following fact holds true:

FACT: Whenever for a point $z \in U_{1} \cap\left(T \backslash T_{2}\right)$ the set ${ }_{z} Q_{z} \cap\left(T_{1} \cup T_{0}\right)$ is the germ of a smooth real curve, then there is a component $\hat{T}$, as defined above, such that ${ }_{z} \hat{T}={ }_{z} Q_{z}$.

Since, however, the sets $A_{z}$ are discrete according to Proposition 2.2, the hypothesis of the "Fact" above can only be satisfied for a finite number of points $z \in U_{1} \cap\left(T \backslash T_{2}\right)$. Therefore, we have altogether shown that $f$ is holomorphically extendable across all points of $U_{1} \cap \partial D$ with the possible exception of a finite number of them. After shrinking $U_{2}$ and $U_{1}$ again, we may therefore assume that $\left(Q_{z^{0}} \cap \partial D\right) \backslash\left\{z^{0}\right\} \subset \Sigma$. Hence, Lemma 12.6 implies also here $z^{0} \in \Sigma$. 
As explained, the sequence of lemmas of this section finishes the proof of Theorem 1.2.

\section{Final remark.}

After Lemmas 12.6 and 12.7, we said we no longer needed the symmetric statements. They do, however, hold.

\section{REFERENCES}

1. M. S. Baouendi, S. Bell, And L. P. Rothschild. Mappings of three-dimensional CR-manifolds and their holomorphic extension, Duke Math. J. 56 (1988), 503-530.

2. M. S. Baouendi, H. Jacobowitz, and F. Treves. On the analyticity of $C R$ mappings, Ann. Math. 122 (1985), 365-400.

3. M. S. Baouendi And L. P. Rothischild. Germs of $C R$ maps between real analytic hyperfaces, Invent. Math. 93 (1988), 481-500.

4. D. BARRETT. Irregularity of the Bergman projection on a smooth bounded domain in $\mathbb{C}^{2}$, Ann. Math. 119 (1984), 431-436.

5. E. Bedford. Proper holomorphic maps, Bulletin AMS 10(2) (1984), 157-175.

6. S. BELl. Analytic hypoellipticity of the $\bar{\partial}-$ Neumann problem and extendability of holomorphic mappings, Acta. Math. 147 (1981), 109-116.

7. S. Bell. Biholomorphic mappings and the overline partial problem, Ann. Math. 114 (1981), 103-133.

8. S. BELL. Proper holomorphic correspondences between circular domains, Comment. Math. Helv. 57 (1982), 532-538.

9. S. Bell and D. Catlin. Regularity of CR mappings, Math. Z. 199 (1988), 357-368.

10. S. Bell and R. Narasimhan. Proper holomorphic mappings of complex spaces, Several Complex Variables (Heidelberg) (Barth, W., Narasimhan, R., eds.), EMS, vol. 69, Springer-Verlag.

11. F. Berteloot. A remark on local continuous extension of proper holomorphic mappings, Contemporary Math. 137 (1992), 79-83.

12. E. Bierstone and P. Milman. Semianalytic and subanalytic sets, Inst. Hautes Etudes Sci. Pub. 67 (1988), 5-42.

13. E. M. Chirka. Complex analytic sets, Kluwer, Dordrecht, 1990.

14. K. Diederich And J. E. Fornæss. Pseudoconvex domains with real analytic boundary, Ann. Math. 107(2) (1978), 371-384.

15. K. Diederich AND J. E. Forness. Biholomorphic maps between certain real analytic domains in $\mathbb{C}^{2}$, Math. Ann. 245 (1979), 255-272.

16. K. Diederich AND J. E. FornÆSS. Applications holomorphes propres entre domaines à bord analytique réel, C. R. Acad. Sci. Paris 307 (1988), 321-324.

17. K. Diederich And J. E. Forness. Proper holomorphic mappings between real-analytic pseudoconvex domains in $\mathbb{C}^{n}$, Math. Ann. 282 (1988), 681-700.

18. K. Diederich, J. E. Forness And Z. Ye. Biholomorphisms in dimension 2, J. Geom. Analysis 4 (1994), 539-552.

19. K. Diederich AND S. WeBster. A reflection principle for degenerate real hypersurfaces, Duke Math. J. 47 (1980), 835-845.

20. F. Forstneric. A survey on proper holomrophic mappings, Proceedings of the Special Year in SCV's at the Mittag-Leffler Institute (Princeton, N. J.) (Fornæss, J. E., ed.), Math. Notes, vol. 38, Princeton University Press.

21. X. HuAng. Regularity of holomorphic correspondences and applications to the mapping problems, Preprint, 1995.

22. H. Lewy. On the boundary behavior of holomorphic mappings, Acad. Naz. Linc. 35 (1977), 1-8. 
23. R. NARAsimhan. Introduction to the theory of analytic spaces, Lecture Notes (New York), vol. 25, Springer- Verlag, New York, 1966.

24. S. I. PINchuk. On the analytic continuation of holomorphic mappings, Math. USSR-Sb. 27 (1975), 375-392.

25. S. I. Pinchuk. Proper holomorphic mappings of strictly pseudoconvex domains, Dokl. Akad. Nauk SSSR 241 (1978), 30-33.

26. J. M. TrÉpreau. Sur le prolongement holomorphe des fonctions $C R$ definies sur une hypersurface reele de classe $C^{2}$ dans $\mathbb{C}^{n}$, Invent. Math. 83 (1986), 583-592.

27. S. Webster. On the mapping poblem for algebraic real hypersurfaces, Inventiones Math. 43 (1977), 53-68.

\section{Klas Diederich}

Department of Mathematics

University of Wuppertal

D-42097 Wuppertal, Germany

\section{Sergey Pinchuk}

Department of Mathematics

Indiana University

Bloomington, Indiana 47405, U.S.A.

Cheliabinsk Federal University of Technology

76 Lenin Avenue

Cheliabinsk 454080, Russia

Received: December 15th, 1995. 